\title{
Modal analysis of the range evolution of broadband wavefields in the North Pacific Ocean: Low mode numbers
}

\author{
Ilya A. Udovydchenkov ${ }^{a)}$ \\ Applied Ocean Physics and Engineering Department, Woods Hole Oceanographic Institution, Woods Hole, \\ Massachusetts 02543 \\ Michael G. Brown \\ Rosenstiel School of Marine and Atmospheric Science, University of Miami, Miami, Florida 33149 \\ Timothy F. Duda \\ Applied Ocean Physics and Engineering Department, Woods Hole Oceanographic Institution, Woods Hole, \\ Massachusetts 02543 \\ James A. Mercer and Rex K. Andrew \\ Acoustics Department, Applied Physics Laboratory, University of Washington, Seattle, Washington 98105 \\ Peter F. Worcester and Matthew A. Dzieciuch \\ Scripps Institution of Oceanography, University of California at San Diego, La Jolla, California 92093 \\ Bruce M. Howe \\ Department of Ocean and Resources Engineering, University of Hawai' i at Manoa, Honolulu, Hawaii 96822 \\ John A. Colosi \\ Department of Oceanography, Naval Postgraduate School, Monterey, California 93943
}

(Received 25 October 2011; revised 19 March 2012; accepted 26 March 2012)

\begin{abstract}
The results of mode-processing measurements of broadband acoustic wavefields made in the fall of 2004 as part of the Long-Range Ocean Acoustic Propagation Experiment (LOAPEX) in the eastern North Pacific Ocean are reported here. Transient wavefields in the $50-90 \mathrm{~Hz}$ band that were recorded on a $1400-\mathrm{m}$ long 40 element vertical array centered near the sound channel axis are analyzed. This array was designed to resolve low-order modes. The wavefields were excited by a ship-suspended source at seven ranges, between approximately 50 and $3200 \mathrm{~km}$, from the receiving array. The range evolution of broadband modal arrival patterns corresponding to fixed mode numbers ("modal group arrivals") is analyzed with an emphasis on the second (variance) and third (skewness) moments. A theory of modal group time spreads is described, emphasizing complexities associated with energy scattering among low-order modes. The temporal structure of measured modal group arrivals is compared to theoretical predictions and numerical simulations. Theory, simulations, and observations generally agree. In cases where disagreement is observed, the reasons for the disagreement are discussed in terms of the underlying physical processes and data limitations. (C) 2012 Acoustical Society of America. [http://dx.doi.org/10.1121/1.4707431]
\end{abstract}

PACS number(s): 43.30.Bp, 43.30.Dr, [AMT]

Pages: 4409-4427

\section{INTRODUCTION}

Understanding of the physics of sound propagation in a fluctuating ocean is important in many applications including underwater communication, navigation, underwater imaging, passive and active target localization, and tomography. Prior to 1989 experimental work in the deep ocean that was designed to elucidate issues relating to the basic physics of forward scattering of sound made use of, for the most part, point-to-point geometries; see Munk et al. (1995) for a review. Beginning in 1989 with the Slice89 experiment (see references in the following text), isolated hydrophones in experimental work were largely replaced by vertical line arrays (VLAs). The work reported here represents a continuation of

\footnotetext{
a) Author to whom correspondence should be addressed. Electronic mail: ilya@whoi.edu
}

the trend toward analyzing wavefields in the deep ocean that are excited by a transient compact source and measured on a VLA. In this paper, measurements made as part of the Long-Range Ocean Acoustic Propagation Experiment (LOAPEX)—see Mercer et al. (2005, 2009)-are analyzed. A modal description of the acoustic wavefield in the 50 to $90 \mathrm{~Hz}$ band is employed. The modal content of the measured LOAPEX wavefields is estimated and compared to numerical simulations and theoretical predictions as a function of propagation range. The modal analysis presented here is made possible because the wavefields in this experiment were measured on a VLA.

Previous VLA-based acoustic wavefield measurements in the deep ocean have been used to investigate a variety of topics. In the Slice89 experiment (Worcester et al., 1994; Cornuelle et al., 1993; Duda et al., 1992), broadband acoustic signals were transmitted from a moored source to a $3-\mathrm{km}$ 
long VLA of 50 hydrophones at a range of $1000 \mathrm{~km}$. A comparison between measured and predicted (mostly with the use of ray theory) broadband acoustic arrival patterns was made, and fluctuations in measured wavefronts were quantified. No modal analysis of the Slice 89 data has been attempted because the receiving array was too sparse to resolve modes in the relevant $200-300 \mathrm{~Hz}$ frequency band. In November 1994, the Acoustic Engineering Test (AET) (Worcester et al., 1999; Colosi et al., 1999; Colosi et al., 2001) was conducted as part of the Acoustic Thermometry of Ocean Climate (ATOC) program. In that experiment, acoustic signals were transmitted from a moored broadband source with $75 \mathrm{~Hz}$ center frequency in the eastern North Pacific Ocean. The wavefield was recorded on a 700-m long 20 element VLA at a distance of $3252 \mathrm{~km}$. Analyses of this data set addressed a number of questions including the accuracy with which gyre- and basin-scale ocean temperature variability can be measured using tomographic methods (Worcester et al., 1999), the vertical resolution that can be obtained at multimegameter range (Worcester et al., 1999) and the influence of smaller-scale processes like internal waves and mesoscale eddies on acoustic variability (Colosi et al., 1999). It was shown (Colosi et al., 1999) that predictions of pulse spread and wave propagations regime using $\Phi-\Lambda$ theory (Flatté et al., 1979) were in serious disagreement with the observations. An alternative ray-based interpretation of the AET measurements was provided by Beron-Vera et al. (2003). Additional ATOC program experiments were performed involving transmissions from Pioneer Seamount to VLAs near both Hawaii and Kiritimati. Modal analyses of those data in the $60-90 \mathrm{~Hz}$ band, with an emphasis on low-order modes, has been presented (Wage et al., 2003; Wage et al., 2005). In the first paper (Wage et al., 2003), a short-time Fourier framework for broadband mode estimation was developed. The second paper (Wage et al., 2005) analyzes mode coherence at long ranges in the ATOC experiment and focuses on modal statistics. Concurrent with the LOAPEX experiment, and utilizing the same VLAs, the SPICEX experiment was performed with a focus on energy in the $200-300 \mathrm{~Hz}$ band. Those data have been analyzed with an eye toward elucidating scattering of energy into deep shadow zones (so-called shadow zone arrivals) by Van Uffelen et al. (2009, 2010); closely related theory is described by Virovlyansky et al. (2011).

The present paper, which focuses on low-order modes in the $50-90 \mathrm{~Hz}$ frequency band, complements these earlier VLA-based studies of deep ocean underwater sound fields. The data analyzed here were measured on a 1400-m long 40 element vertical array centered near the sound channel axis. This array was designed to resolve low-order modes. The wavefields were excited by a ship-suspended source at seven ranges, between approximately 50 and $3200 \mathrm{~km}$, from the receiving array. The focus of the analysis is on the range evolution of broadband modal arrival patterns corresponding to fixed mode numbers, referred to in the following text as "modal group arrivals." Both the second (variance) and third (skewness) moments of broadband distributions of energy with fixed mode number are considered. Attention is limited to the first ten propagating modes. There are two reasons for this limitation. First, only these modes are well resolved by the receiving VLA. Second, the theoretical treatment of scattering (mode coupling) among this group of modes requires special care that is not needed to describe higher order modes; this topic is discussed in detail in the following text. An important aspect of this paper is the integration of our data analysis to the underlying theory of modal group time spreads. The basic elements of the theory of modal group time spreads are described in Udovydchenkov and Brown (2008) and Virovlyansky et al. (2009). An extension of the basic theory that focuses on low-order modes is described in the following text. The extended theory presented here is shown to describe some aspects of the data very well.

Before proceeding it is useful to provide a brief overview of the assets and geometry of the LOAPEX experiment. The experiment was carried out in September and October of 2004 in the eastern North Pacific Ocean. In the experiment, broadband signals in the $50-90 \mathrm{~Hz}$ band were transmitted using a ship-suspended acoustic source. The resulting transient sound fields were recorded on two moored VLAs in close proximity to one another. Transmissions were made at eight stations, seven of which were chosen to lie approximately on a single geodesic path that passes through the receiving array. The geometry of the experiment is shown in Fig. 1 . These seven stations were nominally at ranges of 50 , $250,500,1000,1600,2300$, and $3200 \mathrm{~km}$ from the receiving arrays, thereby providing measurements of the range evolution of the wavefield. The acoustic source was suspended at one or more depths $(350,500$, or $800 \mathrm{~m})$ at each of the seven transmission stations. Signals of different types were transmitted; here only signals consisting of phase-modulated $\mathrm{m}$ sequences are analyzed. The length of each transmission was 1023 digits of the $m$-sequence with one digit equal to two cycles of the carrier frequency. Two center frequencies for broadband transmissions were used: $75 \mathrm{~Hz}$ for $800 \mathrm{~m}$ source depth transmissions and $68.2 \mathrm{~Hz}$ for 350 and $500 \mathrm{~m}$ source depth transmissions. The two receiving VLAs were separated by approximately $5 \mathrm{~km}$ horizontally. One of the VLAs, hereafter referred to as the SVLA (shallow VLA), consisted of 40 hydrophones with $35 \mathrm{~m}$ spacing, covering depths between approximately 350 and $1750 \mathrm{~m}$, centered

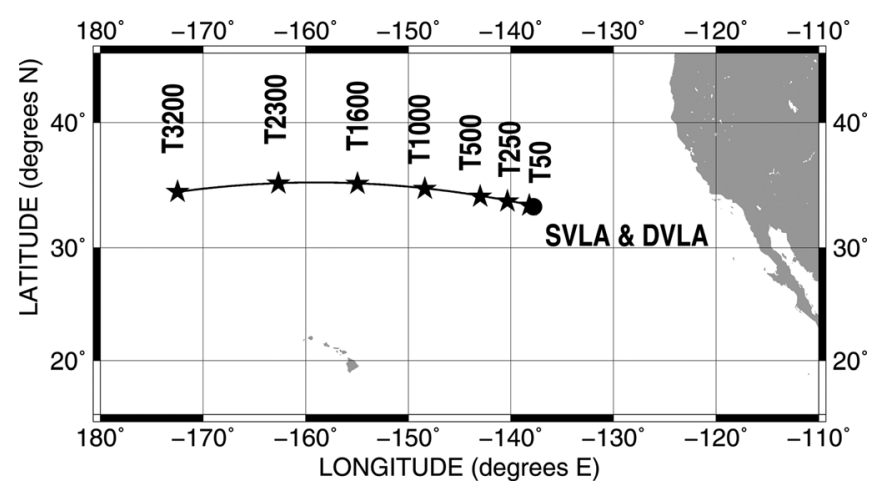

FIG. 1. Geometry of the LOAPEX experiment in the eastern North Pacific Ocean. Two vertical line arrays of hydrophones were deployed at the location denoted "SVLA and DVLA". The source was suspended from the ship at seven stations labeled "T50","T250",...,"T3200" at one or more depths $(350,500$, or $800 \mathrm{~m})$. 
approximately on the sound channel axis. Only measurements from the SVLA are considered in this paper.

The remainder of this paper is organized as follows. The following section provides an overview of the theory of modal group time spreads. Most of this discussion focuses on an extension of the basic theory to correctly treat low mode numbers. In Sec. III, the processing of the LOAPEX data prior to mode-processing is described. In Sec. IV, the construction of modal pulses in LOAPEX suitable for statistical analysis is presented. This includes $75 \mathrm{~Hz}$ center frequency near-axial source (800 m depth) transmissions with transmission ranges up to $500 \mathrm{~km}, 68.2 \mathrm{~Hz}$ center frequency near-axial source (500 m depth) transmissions with transmission ranges of 2300 and $3200 \mathrm{~km}$, and $68.2 \mathrm{~Hz}$ center frequency off-axial source $(350 \mathrm{~m}$ depth) transmissions with transmission ranges up to $3200 \mathrm{~km}$. Some challenges relating to the variations of the signal-to-noise (SNR) ratio in these pulses with transmission range are explained. In Sec. V, mode-processed wavefields and the statistics of modal arrival pulses are compared to full wave numerical simulations and estimates based on a theoretical formulation. A summary of our results is given in Sec. VI.

\section{OVERVIEW OF THE THEORY OF MODAL GROUP TIME SPREADS}

This section contains an overview of theoretical results relating to modal group time spreads (Udovydchenkov and Brown, 2008; Virovlyansky et al., 2009; Makarov et al., 2010). The simplest theoretical results do not correctly treat near-axial (low mode number) scattering. Emphasis is given here to an extension that correctly accounts for low modenumber scattering. The objective of this section is to provide a framework for interpretation of the mode-processed fields described in Secs. IV and V. The phrase "modal group arrival" is used here to describe the contribution to a transient wavefield corresponding to a fixed mode number, and the phrase "modal group time spread" is used to describe the temporal spread of a modal group arrival. It is assumed that the sound speed structure can be decomposed as $c(z)+\delta c(z, r)$, where $c(z)$ is the range-independent background sound speed structure, and the perturbation term $\delta c(z, r)$ is dominated by internal-wave-induced variability. In the LOAPEX environment, this is a good approximation for propagation ranges up to $1000 \mathrm{~km}$; at longer ranges, an adiabatic approximation, which is described in Sec. III, is used to treat the range-dependent background structure. The perturbation term $\delta c(z, r)$ leads to mode coupling, which is treated stochastically. This decomposition of the sound speed structure allows a simple perturbation treatment to be used. The results are based on asymptotic (WKB) mode theory.

\section{A. Basic theory}

At each angular frequency $\omega=2 \pi f$, the acoustic normal modes $\psi_{m}(z)$ satisfy the equation

$$
\frac{d^{2} \psi_{m}(z)}{d z^{2}}+\omega^{2}\left(c^{-2}(z)-p_{m}^{2}\right) \psi_{m}(z)=0,
$$

together with an appropriate pair of boundary conditions. It is assumed that the boundary conditions are such that those conditions together with Eq. (1) define a Sturm-Liouville problem, thereby guaranteeing that the normal modes constitute a complete orthogonal set of functions over the relevant depth domain (the contribution from evanescent modes is assumed to be negligibly small). The modes are real and are assumed to be normalized, $\int\left(\psi_{m}(z)\right)^{2} d z=1$. The constant $p_{m}$ in Eq. (1) is a separation constant. Asymptotically, each mode is associated with a discrete value of the action. For nonreflecting modes (or rays), the action $I$ is defined as

$$
I\left(p_{r}\right)=\frac{1}{\pi} \int_{\check{z}\left(p_{r}\right)}^{\hat{z}\left(p_{r}\right)}\left(c^{-2}(z)-p_{r}^{2}\right)^{1 / 2} d z .
$$

Here $c\left(\hat{z}\left(p_{r}\right)\right)=c\left(\check{z}\left(p_{r}\right)\right)=1 / p_{r}$, where $\check{z}$ and $\hat{z}$ are lower and upper turning points, respectively, and $p_{r}$ is the horizontal component of the slowness vector. In the background environment, both $p_{r}$ and $I$ are constants following rays and modes. For nonreflecting modes, the modal quantization condition, which defines $I=I(m, \omega)$ where $m$ is mode number, is

$$
\omega I\left(p_{m}\right)=m+\frac{1}{2}, \quad m=0,1,2, \ldots
$$

This equation defines a discrete set of $p_{r}$ values, denoted $p_{m}$. A simple, but important, observation is that both $m$ and $I$ are nonnegative. Our use of Eq. (3) involves a simple form of ray-mode duality between the continuous ray variable $I$ and the discrete modal variable $m$. The range and travel time of a ray double loop are $R\left(p_{r}\right)=-2 \pi d I / d p_{r}$ and $T\left(p_{r}\right)=2 \pi I\left(p_{r}\right)$ $+p_{r} R\left(p_{r}\right)$, respectively. Modal energy at range $r$ arrives at time $t=S_{g}(m, \omega) r$ where $S_{g}$ is the group slowness,

$$
S_{g}\left(p_{r}\right)=\frac{T\left(p_{r}\right)}{R\left(p_{r}\right)} .
$$

Modal dispersion is controlled by the waveguide invariant,

$$
\beta(m, \omega)=-\frac{\partial S_{g}}{\partial p_{r}} .
$$

Asymptotically $\beta$ depends only on $p_{r}$; the quantization condition, Eq. (3), picks out the relevant $p_{m}$ values and, in turn, the dependence of $\beta$ on $m$ and $\omega$. Consistently with WKB approximation, $\beta$ depends only on the ratio $(m+1 / 2) / \omega$. Therefore, the change of frequency in the quantization condition (3) does not change the structure of the $\beta(m)$ dependence, but only requires linear rescaling of the mode number axis.

There are three contributions to modal group time spreads, which combine approximately in quadrature (Virovlyansky, 2006; Udovydchenkov and Brown, 2008; Virovlyansky et al., 2009; Makarov et al., 2010),

$$
\Delta t_{m}(r)=\sqrt{\Delta t_{b w}^{2}+\Delta t_{d}^{2}+\Delta t_{s}^{2}} .
$$

Here 


$$
\Delta t_{b w}=(\Delta f)^{-1},
$$

where $\Delta f$ is the frequency bandwidth of the acoustic source,

$$
\Delta t_{d}=-\frac{2 \pi I_{c} \beta\left(I_{c}\right)}{R\left(I_{c}\right) f_{c}} r \Delta f
$$

is the deterministic dispersive contribution, where $f_{c}$ is the center frequency of the acoustic source and $I_{c}$ is defined by the condition $2 \pi f_{c} I_{c}=m+1 / 2$. For large mode numbers, the scattering-induced (associated with mode coupling) contribution to a modal group time spread is

$$
\Delta t_{s}=4 \pi^{3 / 2} \frac{\left|\beta\left(I_{0}\right)\right|}{R\left(I_{0}\right)}\left(\frac{B}{3}\right)^{1 / 2} r^{3 / 2} .
$$

Here $B$ is the diffusivity of action defined by the condition $\overline{\left(I(r)-I_{0}\right)^{2}}=B r$, where $I_{0}=I(r=0)$, and the overline denotes expected value (or an ensemble average). A simple derivation of Eq. (9) (Udovydchenkov and Brown, 2008) makes use of a discrete scattering model in which the action associated with a ray or mode experiences a sequence of independent random kicks. Note that as energy diffuses in action in the ray description, it also diffuses in mode number, taking discrete steps, according to the quantization condition (3). The derivation of Eq. (9) requires $\overline{I(r)}=I_{0}$; thus both $I_{c}$ and $I_{0}$ lie at the center of fixed- $m$ action distributions. For most purposes, it is unnecessary to notationally distinguish between these quantities. All four measures of time spread [(6)-(9)] characterize full widths of temporal distributions at the $e^{-\pi / 4}$ amplitude level, which is approximately the same as the half-amplitude level. Consistent with this choice of $\Delta t$, assuming that distributions are approximately Gaussian, the variance of each amplitude distribution is $(\Delta t)^{2} / 2 \pi$ (for each of the four choices of $\Delta t$ ). For correct comparison of theoretical predictions with numerical simulations and data, it is important to chose the bandwidth $\Delta f$ in Eqs. (7) and (8) in a consistent way. Here we have chosen $\Delta f=f_{c} / 4$. This choice is justified in the following text and discussed together with observations made regarding the spectrum of the LOAPEX acoustic source.

\section{B. Low mode numbers}

The principal limitation of Eq. (9) is that the derivation of this expression does not invoke the constraint that $I \geq 0$. Because of that limitation, the validity of Eq. (9) is limited to $I_{0} \gg \sqrt{B r}$, i.e., to large mode numbers. The focus of this paper is on low mode numbers, so an alternate approach to the estimation of $\Delta t_{s}$ is required. A framework for addressing this problem, which we now review, was provided in Virovlyansky (2006) and Virovlyansky et al. (2007, 2009) using results from the theory of stochastic differential equations. It was shown that, at ranges in excess of a few complete ray cycle distances, multiple weak uncorrelated scattering events lead to a particularly simple Fokker-Planck equation, which governs the evolution in range of the probability density function of action,

$$
\frac{\partial P}{\partial r}=\frac{\partial}{\partial I}\left(\frac{B}{2} \frac{\partial P}{\partial I}\right)
$$

Let $P\left(I, r \mid I_{0}\right)$ denote the fundamental solution to this equation, which satisfies the initial $(r=0)$ condition $P\left(I, 0 \mid I_{0}\right)$ $=\delta\left(I-I_{0}\right)$. Given knowledge of $P\left(I, r \mid I_{0}\right)$, statistics as a function of range of any function of $I$, including modal group time spreads, can be computed. To compute the statistics of modal group arrivals, one makes use of the simple result that the arrival time of energy with action history $I(r)$ is $T(r)=\int_{0}^{r} S_{g}\left(I\left(r^{\prime}\right)\right) d r^{\prime}$ together with the definition of the expected value of the action $\bar{I}=\int I P\left(I, r \mid I_{0}\right) d I$ and a Taylor series expansion of $S_{g}(I)$ around the expected value of the action, $S_{g}(I) \approx S_{g}(\bar{I})+S_{g}^{\prime}(\bar{I})(I-\bar{I})=S_{g}(\bar{I})+(2 \pi \beta(\bar{I}) / R(\bar{I}))$ $(I-\bar{I})$. The expected value of $T$ is

$$
\bar{T}=S_{g}(\bar{I}) r+\frac{2 \pi \beta(\bar{I})}{R(\bar{I})} \int_{0}^{r} d r^{\prime} \int d I(I-\bar{I}) P\left(I, r^{\prime} \mid I_{0}\right)=S_{g}(\bar{I}) r
$$

where we have used $\int P\left(I, r \mid I_{0}\right) d I=1$. The expected squared spread in $T$ is

$$
\overline{(T-\bar{T})^{2}}=\left(\frac{2 \pi \beta(\bar{I})}{R(\bar{I})}\right)^{2} \sigma_{t}^{2},
$$

where

$$
\begin{aligned}
\sigma_{t}^{2}= & \int_{0}^{r} d r_{1} \int_{0}^{r} d r_{2} \int d I_{1}\left(I_{1}-\bar{I}\left(r_{1}\right)\right) \\
& \int d I_{2}\left(I_{2}-\bar{I}\left(r_{2}\right)\right) P\left(I_{1}, I_{2}, r_{1}, r_{2} \mid I_{0}\right) .
\end{aligned}
$$

Here $P\left(I_{1}, I_{2}, r_{1}, r_{2} \mid I_{0}\right)$ is a joint probability density function, which, in turn, depends on a conditional probability density function. (Recall that $P(a \cap b)=P(a ; b) P(b)=P(b ; a) P(a)$ where $a \cap b$ denotes the intersection of $a$ and $b$, and $P(a ; b)$ is the conditional probability of $a$ given $b$ ). An expression for $P\left(I_{1}, I_{2}, r_{1}, r_{2} \mid I_{0}\right)$ that was used for theoretical estimates of modal group time spreads is derived in the Appendix. Before presenting results for the low mode number problem, we note that the preceding results can be used to derive Eq. (9). To see this, first note that with $B$ constant the fundamental solution to the Fokker-Planck equation (10) on an $I$-domain extending from $-\infty$ to $+\infty$ is a Gaussian with the mean $I_{0}$ and the variance $B r$,

$$
P\left(I, r \mid I_{0}\right)=\frac{1}{\sqrt{2 \pi B r}} e^{-\frac{\left(I-I_{0}\right)^{2}}{2 B r}}
$$

From Eq. (14) it follows that $\bar{I}=I_{0}$ and $\bar{T}=S_{g}\left(I_{0}\right) r$. Using a well-known expression (Beichelt, 2006, pp. 357, 358, 387-389) for the joint normal distribution, the square root of the rhs of Eq. (12) reduces to $(2 \sqrt{\pi})^{-1}$ times the rhs of Eq. (9) - after replacing $\bar{I}$ by $I_{0}$. (The factor of $2 \sqrt{\pi}$ is required for consistency of spread estimates according to our definitions of $\Delta t$ ). These observations provide the link between Eqs. (9) and (12) for large mode numbers $\left(I_{0} \gg \sqrt{B r}\right)$. 
Equations (10)-(13) also apply to near-axial scattering, corresponding to small mode numbers, for which the condition $I \geq 0$ is a critically important restriction. A foundation for treating this problem is the observation by Virovlyansky (Virovlyansky et al., 2007; Virovlyansky, 2006; Virovlyansky et al., 2006) that the Fokker-Plank equation (10) admits an exact solution in the presence of a reflecting boundary at $I=0$. The fundamental solution is

$$
P\left(I, r \mid I_{0}\right)=\frac{1}{\sqrt{2 \pi B r}}\left\{e^{-\frac{\left(I-I_{0}\right)^{2}}{2 B r}}-e^{-\frac{\left(I+I_{0}\right)^{2}}{2 B r}}\right\}
$$

With this $P\left(I, r \mid I_{0}\right)$, some integrals that describe statistical quantities of interest can be evaluated (keeping in mind that the relevant $I$-domain is $[0, \infty))$. For example,

$$
\overline{I(r)}=\sqrt{\frac{2 B r}{\pi}} e^{-\frac{I_{0}^{2}}{2 B r}}+I_{0} \times \operatorname{erf} \frac{\mathrm{I}_{0}}{\sqrt{2 \mathrm{Br}}}
$$

and

$$
\overline{(I(r))^{2}}=I_{0}^{2}+B r
$$

so the variance is

$$
\sigma_{I}^{2}(r)=\overline{I^{2}}-\bar{I}^{2}=I_{0}^{2}+B r-\left(\sqrt{\frac{2 B r}{\pi}} e^{-\frac{I_{0}^{2}}{2 B r}}+I_{0} \times \operatorname{erf} \frac{\mathrm{I}_{0}}{\sqrt{2 \mathrm{Br}}}\right)^{2} .
$$

Some important properties of these expressions are: (1) $\overline{I(0)}=I_{0}$; (2) $\sigma_{I}^{2}(0)=0$; and (3) $\overline{I(r)}$ increases monotonically with increasing $r$ (so there is a preference, on average, for mode coupling toward higher mode numbers). These properties are illustrated in Fig. 2. Equation (13) remains valid with the modified $P\left(I, r, \mid I_{0}\right)(15)$ (provided the appro- priate joint probability density function is used). Unfortunately, it does not appear to be possible to evaluate or otherwise simplify those integrals.

Figure 2 shows numerical simulations designed to illustrate the difference between time spreads based on Eqs. (14) and (15). As noted in the preceding text, if $\sigma_{t}^{2}$ was evaluated using Eq. (14), then it would be equal to $(B / 3) r^{3}$. Thus the difference between $\sigma_{t}^{2}$ and $(B / 3) r^{3}$ can be attributed to the difference between near-axial/low mode number (15) and offaxial/high mode number (14) scattering. The right panel of Fig. 2 shows a family of $\sigma_{t}(r)$ curves (normalized by $r^{3 / 2}$ ), each corresponding to an initial condition in which energy is contained in a single mode $m_{0}$. Those curves are seen to be bounded by two asymptotes. For small $r, \sigma_{t}^{2} \simeq B / 3$; for large $r, \sigma_{t}^{2} \simeq B / 6$. Small/large $m_{0}$ values rapidly/slowly approach the large $r$ asymptote. This behavior can be explained by noting that for $m_{0}$ large and $r$ small, there is negligible energy near the $I=0$ "boundary," whereas for $m_{0}$ small and $r$ large, the $I=0$ "boundary" is felt by essentially all of the energy present. While the qualitative behavior shown in Fig. 2 is both insightful and important, that figure is of limited value in terms of describing modal group arrivals during LOAPEX. The principal limitations of Fig. 2 are that those simulations do not account for: (1) the scattering-induced travel time bias, i.e., the fact that in general for low mode numbers $\bar{T} \neq S_{g}\left(I_{0}\right) r$; and (2) the fact that experimentally many mode numbers are excited at $r=0$. Also note that the discussion in the preceding text including the validity of Eq. (12) is limited to the case when $\beta(\bar{I}) / R(\bar{I})$ does not vary significantly along the propagation path. Under typical experimental conditions, $R(\bar{I})$ is not expected to vary rapidly within a small group of modes, but variations of $\beta(\bar{I})$ may be significant.

\section{A simple numerical model}

An unfortunate conclusion to be drawn from the preceding discussion is that the constraint $I \geq 0$ precludes, so far as
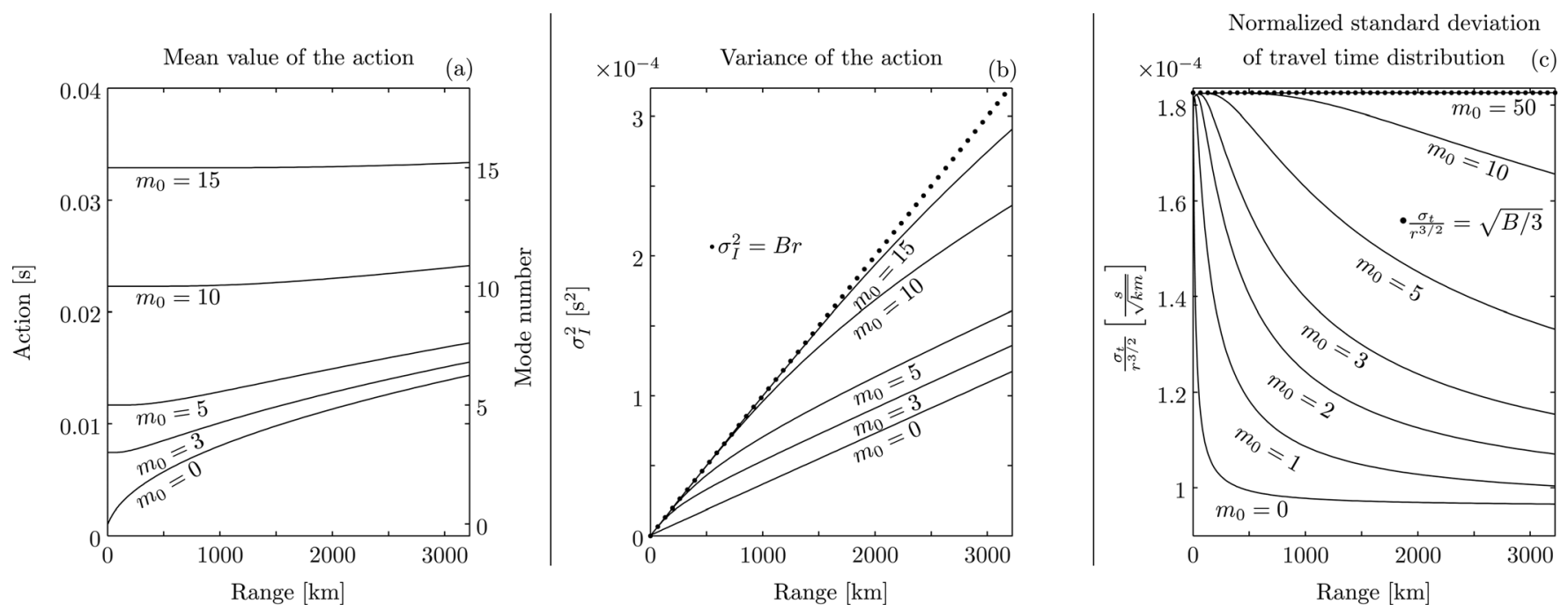

FIG. 2. (Left panel) Mean value of the action distribution as a function of range for several initial values of mode number $m_{0}$ computed using Eq. (16). Significant energy redistribution toward higher mode numbers is observed for $m_{0} \lesssim 10$. (Middle panel) Variance of action as a function of range for several values of $m_{0}$ computed using Eq. (18). The high mode number asymptotic result, corresponding to linear growth of variance, is shown as a dotted line. (Right panel) $\sigma_{t}$ (defined in the text) divided by $r^{3 / 2}$ for different initial values of mode number $m_{0}$ at $75 \mathrm{~Hz}$ computed using Eq. (13). The limiting behavior at $I_{0} \gg \sqrt{B r}$ is shown as a dotted line. 
we are aware, derivation of an analytical expression that accurately describes modal group time spreads for small $\mathrm{m}$. This challenge has been addressed by developing a very simple numerical model that accounts for all of the theoretical elements that were just described. Predictions based on the simple model are described throughout the remainder of this paper as "theory-based model predictions." Such theorybased model predictions are compared in the following text to estimates of modal group time spreads derived from LOAPEX observations and from full wave numerical simulations. Here the simple theory-based model is described.

Like the estimate (9), the key assumption underlying the treatment of the scattering-induced contribution to modal group time spreads is the diffusive spreading of energy in action. The model makes use of an ensemble of solutions to the equation $T(r)=\int_{0}^{r} S_{g}\left(I\left(r^{\prime}\right)\right) d r^{\prime}$, where $I(r)$ is a random walk with a reflecting $(I \geq 0)$ boundary. Because of the connection between ray and modal description through the quantization condition (3), this equation allows estimation of a modal energy arrival time, which is the same as the travel time of the corresponding ray arrival. Random walk simulations with a reflecting boundary were previously used by Virovlyansky (Virovlyansky, 2006; Virovlyansky et al., 2007) to investigate low-mode scattering. In our simulations, both the group slowness structure $S_{g}(I)$ and the diffusivity $B$ are measurement-based estimates. Many (typically millions) action histories were used in each simulation. Initial conditions were chosen to mimic experimental conditions. To approximately account for non-uniform excitation of modal amplitudes by the compact source, the number of initial conditions for each value of $I_{0}$ was taken to be $\left[w_{m} N\right]$ (square brackets denote the nearest integer; $N=50000$ was chosen for all simulations), where $w_{m}=\left(\psi_{m}\left(z_{s}, f_{c}\right) / \max \left|\psi_{m}\left(z_{s}, f_{c}\right)\right|\right)^{2}$ is proportional to the square of the modal function at the source depth and at the carrier frequency; the arbitrary normalization constant in this formula was chosen so that $w_{m}=1$ for the modal function with the largest amplitude at the source depth and at the carrier frequency. This approximation to the initial condition is consistent with the ray density (number of initial rays assigned to each $I_{0}$, and hence to each $m$ ) being proportional to the wavefield intensity. Each ray in the ensemble undergoes an independent random walk (with reflection at $I=0$ ). At the final range, all arrival times (or the corresponding values of $S_{g}$ ) are split into bins and a histogram representing the density of rays, which is proportional to the relative wavefield intensity, as a function of arrival time and action is computed. This procedure gives a prediction of the temporal distribution of scattered energy at the final range as a function of $I$. By making use of the quantization condition (3), evaluated at the center frequency of the transmission, dependence on $I$ can be converted into dependence on mode number $m$ resulting in a prediction of the temporal distribution of scattered energy as a function of mode number. Note that this procedure estimates modal amplitudes at the source only up to a constant, and it does not account for variations of amplitudes of modal excitation with frequency at the source location. Absolute amplitudes of arrivals can not be computed using this method, but some statistics of modal arrivals can be estimated.
To account for the deterministic dispersive and reciprocal bandwidth contributions to modal group time spreads, $\Delta t_{d}$ and $\Delta t_{b w}$, two perturbations are added to each scattered $t$ value at the final range. These perturbations are randomly drawn from Gaussian distributions the variances of which are $\left(\Delta t_{d}\right)^{2} / 2 \pi$ and $\left(\Delta t_{b w}\right)^{2} / 2 \pi$. [The reason for including the factors of $2 \pi$ is explained following Eq. (9).] If all of the $I_{0}$ values that contribute to a particular $m$ satisfy $I_{0} \gg \sqrt{B r}$ and if $S_{g}(m)$ is a slowly varying function, the procedure that we have just described gives, to an excellent approximation, Gaussian distributions with mean $t=S_{g}(m) r$ and variance $\left(\Delta t_{m}\right)^{2} / 2 \pi$ (6). In other words, the procedure that we have described reduces to Eqs. (6)-(9) as a special case. We have used this special case as a test of our numerical algorithm. If the condition $I_{0} \gg \sqrt{B r}$ is not satisfied, the simulated distributions are generally non-Gaussian, and their first moments may deviate significantly from $t=S_{g}(m) r$.

\section{LOAPEX DATA PROCESSING}

Recall that in LOAPEX transmissions at multiple depths and ranges were recorded on the SVLA. On each hydrophone on the SVLA linearly scaled acoustic pressure was recorded in a 2-byte integer format. Specially coded signals (m-sequences) were transmitted to achieve high SNR, so the recorded data was match-filtered against the reference sequence. Several additional timing corrections, mooringmotion corrections, and source motion corrections were applied. Also, note that environmental data are needed to construct simulated acoustic wavefields and to compute the modes that are used to perform mode filtering. After mode filtering is performed, the statistics of modal group arrivals are computed. In this section, some details are provided about those processing steps that are performed prior to mode filtering.

\section{A. Environmental data}

Mode filtering is most naturally done using modes constructed using the sound speed profile at the SVLA [recall Eq. (1)]. Sound speed profiles were constructed from measurements made on the SVLA. There were 10 MicroCAT (SBE 37) sensors (Sea-Bird Electronics, Inc., 2003a) that measured temperature and salinity and 14 MicroTemp (SBE 39) sensors (Sea-Bird Electronics, Inc., 2003b) that measured only temperature. Those sensors were attached to the SVLA mooring at known depths spanning the water column between about 150 and $2875 \mathrm{~m}$. Linear interpolation on a temperature-salinity diagram was used to infer missing values of salinity at depths of MicroTemp sensors. Because the deepest MicroCAT sensor was only at about $900 \mathrm{~m}$, measurements of salinity from deep CTD (conductivity-temperature-depth) casts at transmission stations T50 and T250 were used to augment missing salinity profiles in deep water. Note that reconstruction of salinity profiles below $2875 \mathrm{~m}$ does not influence the modal analysis because those depths are not covered by the SVLA. The Del Grosso (Del Grosso, 1974) formula was used to compute sound speed profiles. These profiles were updated approximately every $7 \mathrm{~min}$ throughout the entire experiment. Modes were computed in 
every profile using KRAKEN normal mode code (Porter, 1991; Jensen et al., 2000). Note that eigenmodes are frequencydependent, so they have to be computed for each frequency within the band of interest. We used 256 frequencies covering the bandwidth from 37.5 to $112.5 \mathrm{~Hz}$. Linear interpolation (across frequency) was used to construct eigenmodes at intermediate frequencies when needed.

Ideally, to compare results of data processing with numerical models and theoretical predictions, one needs to know the environment along the entire propagation path. During the experiment, seven deep CTD casts were made (see Fig. 3, left panel), one at each transmission station. In addition, underway CTD (UCTD) measurements were made in the upper $350 \mathrm{~m}$ approximately between T50 and T1600 transmission stations. A set of full water column profiles was derived by Lora Van Uffelen (personal communication) from the UCTD measurements by objectively mapping them onto the Levitus World Ocean Atlas 2005 database. However, for the analysis presented in this paper, we assume that the sound speed profile along the propagation path can be represented as a range-independent background profile $c(z)$ on top of which a range-dependent sound speed perturbation $\delta c(z, r)$, due to internal waves, for example, is superimposed. This assumption holds well between about T1000 and SVLA. Note also in this regard that the existing theory described in Sec. II does not account for strong mesoscale variability in the background sound speed structure. While full wave numerical simulations can be performed in environments with background range-dependence, we decided to use the same environmental structure [background $c(z)$ plus perturbation $\delta c(z, r)]$ in those simulations to make interpretation and comparison of results easier. Therefore we have constructed four different background profiles, shown in the middle panel of Fig. 3 and labeled C50, C250, C500, and C1000. These are range-averaged profiles from the set derived by Van Uffelen over the respective range. As described in the following text, somewhat better agreement between data and full wave numerical simulations is achieved for transmissions from T50 if a profile constructed from the Seabird measurements (at the SVLA) is used as the background profile for those simulations. This profile (time-averaged over the entire time of transmissions from T50 station) is labeled C50SB in the middle panel of Fig. 3 and is clearly different from C50 constructed by objective mapping. For theoretical predictions and full wave numerical simulations, we use C50SB profile for transmissions from the station T50 and will use the range-averaged UCTD-based profiles C250, C500, and C1000 for transmissions from T250, T500, and T1000 stations, respectively.
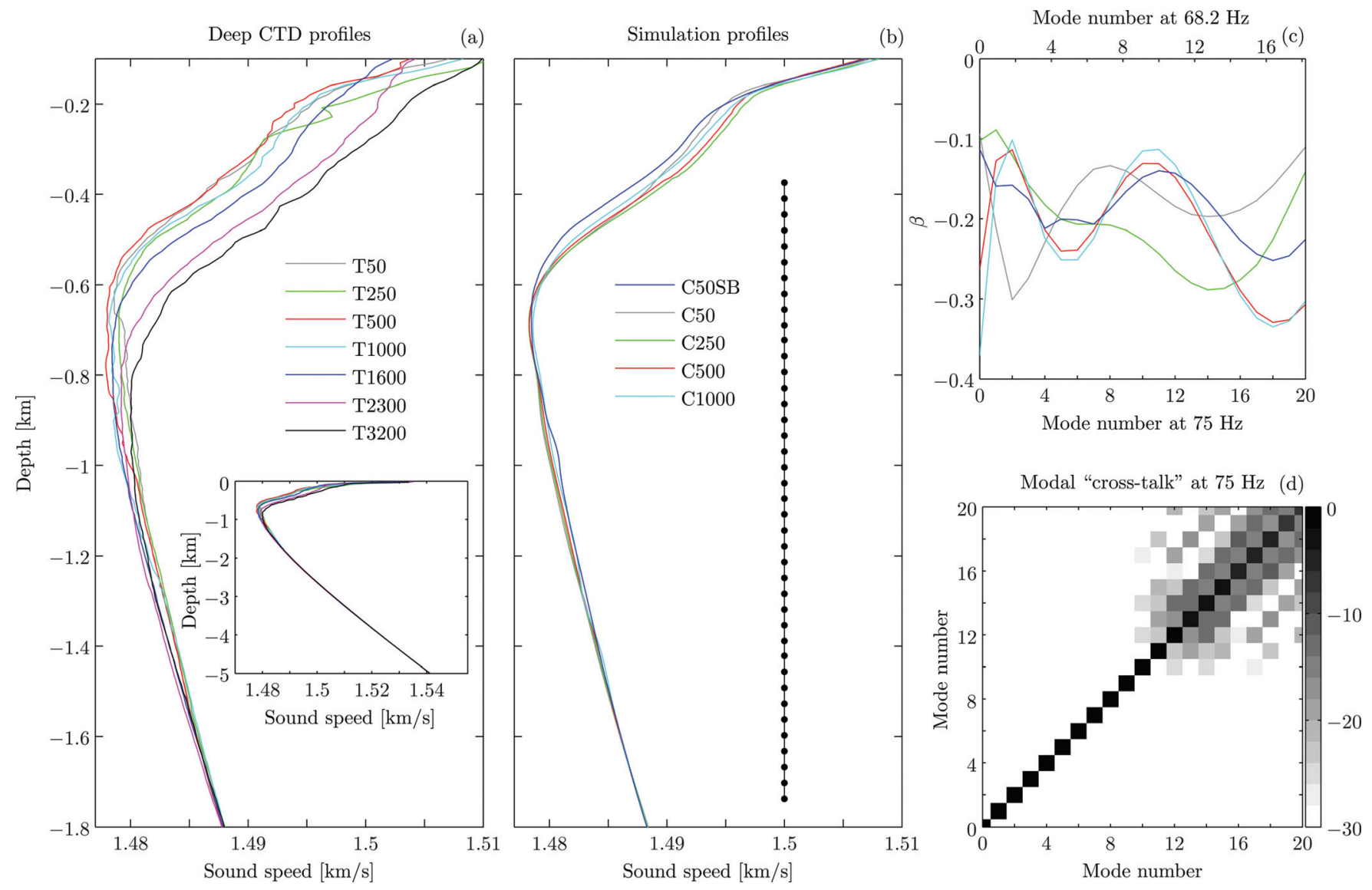

FIG. 3. (Left panel) Sound speed profiles computed from deep CTD casts made at seven LOAPEX stations. (Middle panel) Sound speed profiles used as background $c(z)$ for numerical simulations and theoretical predictions. C50SB profile was used instead of C50 profile for simulations and theoretical predictions for transmissions from T50. The SVLA array geometry is also shown. (Upper right panel) Waveguide invariant dependence on mode number at 75 and $68.2 \mathrm{~Hz}$ for profiles shown in the middle panel. (Lower right panel) Modal "cross-talk" diagram computed in C50SB profile at 75 Hz using time-averaged hydrophone depths. 
Finally, note that the deep CTD measurements made along the LOAPEX track clearly show strong range dependence past $1000 \mathrm{~km}$ range, but the number of measurements is insufficient to construct a mesoscale-resolving range-dependent environment. To approximately account for this background range dependence, we make use of an adiabatic approximation. Under this approximation, we replace the true source depth with the effective source depth in the C1000 environment that lies on the same action surface as the true source depth in the correct local environment (T1600, T2300, or T3200). Then the same modes are excited in the $\mathrm{C} 1000$ environment by the effective source that are excited by the true source in the local environment. This leads to the following transformed depths: at T1600, from 350 to $301 \mathrm{~m}$; at $\mathrm{T} 2300$ from 350 to $235 \mathrm{~m}$ and from 500 to $406 \mathrm{~m}$; at T3200 from 350 to $209 \mathrm{~m}$ and from 500 to $379 \mathrm{~m}$. These corrections are important for quantifying the scattering-induced excitation of low-order modes when the wavefield is excited by the off-axial source.

As described in Sec. II, theory predicts that deterministic dispersive and scattering-induced contributions to modal group time spreads are proportional to the waveguide invariant $\beta(m, f)$. Figure 3 shows $\beta(m, f)$ curves for the two center frequencies used in the experiment: 75 and $68.2 \mathrm{~Hz}$ in each of the environments considered. To construct these curves we computed $S_{g}(m)$ in each profile using Eqs. (3) and (4) and then used a high order (15-20) polynomial to obtain a good fit to $S_{g}(m)$. After that, $\beta(m)$ was computed using Eq. (5). These curves show that although $\beta(m)$ is different in the different background profiles that we have constructed, for all modes of interest $(m=0,1, \ldots, 9) \beta \approx-0.2$. Unfortunately, the fact that only first 10 modes are resolved imposes a great constraint on verifying the importance of $\beta(m)$ in the estimation of modal group time spreads. In all of the environments that we have constructed, $\beta(m)$ is close to zero for mode numbers between approximately 25 and 30; consistent with theoretical predictions, full wave numerical simulations in these environments reveal very small time spread for those modes. Unfortunately the LOAPEX observations do not allow those modes to be resolved to test the theory.

\section{B. Measured acoustic wavefields}

In this subsection, the construction of acoustic wavefields, i.e., measured absolute intensities (resulting from the transmission of a short pulse equal in duration to one digit of the $m$-sequence) as functions of absolute arrival time and depth is described. For mode filtering, the data has to be sampled on a vertical line array at a single range from the acoustic source. The nominal SVLA ranges are 44.714, 244.7, 484.7, 984.7, 1594.7, 2294.7, and $3194.7 \mathrm{~km}$ for T50,.., T3200, respectively. Six phase (timing) corrections must be applied to the measurements. The first timing correction that was applied is $1 \mathrm{~ms}$ and is due to the first sample occurring $1 \mathrm{~ms}$ after the nominal reception time. The second correction is $3.4 \mathrm{~ms}$ and accounts for the total instrumentation processing delay. The third correction accounts for clock drift (which was measured). These corrections are trivial to apply. The fourth correction is the source delay. This correction is due to the phase response characteristics of the source. This correction has been estimated as described in Chandrayadula (2009) by comparing peaks of wavefield intensities at each depth for all T50 $800 \mathrm{~m}$ source transmissions for the two early stable arrivals with ray travel time predictions made using EIGENRAY code (Dushaw and Colosi, 1998). The average among all T50 $75 \mathrm{~Hz} 800 \mathrm{~m}$ transmissions source delay was estimated to be $14.7 \mathrm{~ms}$ and is in good agreement with the number reported in Chandrayadula (2009). It is assumed that the source delay is constant for all transmissions. The fifth correction is due to the motion of the source and therefore, deviation of the actual transmission ranges from the nominal. The source position was updated every period of the transmitted $m$-sequence $(27.28$ and $30 \mathrm{~s}$ for signals with 75 and $68.2 \mathrm{~Hz}$ center frequencies, respectively). The processing gain associated with Doppler corrections for source motion was estimated to be a few tenths of a decibel for most transmissions, and infrequently approximately a half a decibel (Andrew et al., 2010). Those corrections were not implemented in the results presented here. Finally, the most difficult correction to apply accounts for receiver mooring motion. Mooring motion data for all 40 hydrophones was recorded approximately once an hour throughout the entire experiment. Unfortunately, these records contain many gaps, and in some instances only the upper 20 hydrophones or the lower 20 hydrophones were navigated. The data that correspond to transmission times when no navigation data are available for either part of the array $(\mathrm{T} 100075 \mathrm{~Hz} 800 \mathrm{~m}$ source transmissions and part of the T1000 $68.2 \mathrm{~Hz} 350 \mathrm{~m}$ transmissions) are not analyzed in this paper. At times when the navigation data are available for only half of the array, an empirical orthogonal function (EOF) fit was used (Emery and Thomson, 2004). This method was previously applied to the LOAPEX data set by Chandrayadula and Wage (Chandrayadula and Wage, 2008). We have used a slight variation of this method, as described in Lin et al. (2010), that uses a damped (rather than truncated) EOF expansion. Each time series was demeaned before applying the EOF analysis to avoid most of the energy being assigned to the EOF representing the mean state.

There are two types of errors contributing to the resulting wavefields associated with mooring motion corrections. First, there are uncertainties in the mooring position estimates. These errors are known from the navigation solutions, and they were used to estimate position uncertainties when EOF fitting was required. It was estimated that typically these errors in displacements do not exceed a few meters (usually $3 \mathrm{~m}$ or less) for all hydrophones. The second source of errors results from the approximation used to correct phases of wavefields due to mooring displacement from the nominal range. The following argument shows that these errors are negligible. Assuming a typical horizontal mooring displacement of $50 \mathrm{~m}$ from the nominal position, and a maximum perturbation to the phase slowness (among the first 10 modes and between 55 and $95 \mathrm{~Hz}$ computed from hydrographic data using KRAKEN) of $\Delta p_{r}=0.0016 \mathrm{~s} / \mathrm{km}$, the estimated phase error is $\Delta \varphi=2 \pi f \Delta p_{r} r \approx 0.04 \ll 2 \pi$. In this analysis, the mean value of the phase slowness among the 
first 10 modes at the center frequency computed from Seabird data was used for mooring motion corrections.

To compute absolute intensities the raw data recorded in "counts" was converted to Pascals using the linear scaling factor $(963 \mu \mathrm{Pa} /$ count). Care is required to keep track of processing gain from the match-filtering procedure and other processing steps. In addition one needs to know the source level for every transmission. Source level estimates were taken from the cruise report (Mercer et al., 2005). The source level varied from transmission to transmission as described in Appendix 2 of that report. When the source level was not documented it was assumed to be proportional to the $10 \times \log _{10}$ (voltage $\times$ current) and missing values were filled in using linear interpolation.

An important decision in the construction of acoustic wavefields is how much coherent averaging of receptions should be done to improve SNR. Coherent processing gain of successive receptions typically varies between approximately $10 \times \log _{10}(n)$ (where $n$ is the number of transmissions being averaged) for short times and at shorter ranges, and near-zero for long times and at longer ranges. The results presented here were obtained by averaging receptions over $5 \mathrm{~min}$. (which corresponds to 11 transmissions at $75 \mathrm{~Hz}$ and 10 transmissions at $68.2 \mathrm{~Hz}$ ) for transmissions from stations up to T1600 (and confirming that in most cases the gain of $10 \times \log _{10}(n)$ was achieved) and over $18 \mathrm{~min}$. (36 transmissions) for transmissions from stations T2300, and T3200 (however, observing that often the deviation from the $10 \times \log _{10}(n)$ gain regime is significant). Those averaging times were chosen in part for convenience (averaging was done over one data file). We did not attempt to find optimal averaging times for every transmission. Questions related to the loss of coherence deserve a separate study, but they are outside of the scope of this paper. Coherent stacking was done after mode processing individual receptions.

\section{Numerically simulated acoustic wavefields}

To construct numerically simulated wavefields for comparisons with data and theory, the RAM acoustic propagation model (Collins and Westwood, 1991; Collins, 1993) was used. Internal-wave-induced sound speed perturbations were modeled using the procedure described by Colosi and Brown (Colosi and Brown, 1998). Range-averaged buoyancy frequency $N(z)$ and acoustic fluctuation strength parameter $\mu(z)=-(\rho / c) \Delta c_{\text {pot }} / \Delta \rho_{\text {pot }}$ (where $c_{\text {pot }}$ and $\rho_{\text {pot }}$ are potential sound speed and potential density, respectively) profiles were estimated from hydrographic data collected during the LOAPEX. Other internal wave field parameters used were $E=E_{G M}$ (the nominal Garrett-Munk strength parameter), $j_{\max }=30, k_{\max }=2 \pi / 400 \mathrm{~m}$, and $k_{\min }=2 \pi / 3276.8 \mathrm{~km}$. The internal wave perturbation field was sampled in range every $100 \mathrm{~m}$. The RAM model allows computation of absolute transmission loss, so, with knowledge of the source level, absolute values of wavefield intensity can be computed and compared with the data. The acoustic source spectrum was chosen to have the shape of a Hanning window with peak at the center frequency and zeros at $f_{0}-f_{0} / 4$ and $f_{0}+f_{0} / 4$ (consistent with the discussion above $\left.\Delta f \approx f_{0} / 4\right)$. This choice will be discussed in Sec. V. Satellite-derived bathymetry (Smith and Sandwell, 1997) was used with the bottom properties similar to those given in Stephen et al. (2009). The bottom has three "bathymetry-following" layers, i.e., their thickness is given relative to the seafloor depth. The top layer of sediment is $40 \mathrm{~m}$ thick with compressional velocity of $1.6 \mathrm{~km} / \mathrm{s}$, linear gradient of density from 1.35 to $1.41 \mathrm{~kg} / \mathrm{m}^{3}$, and attenuation of $0.2 \mathrm{~dB} /$ wavelength. The second layer is $1000 \mathrm{~m}$ thick with compressional velocity linearly increasing from 4 to $5.4 \mathrm{~km} / \mathrm{s}$, a linear gradient of density from 2.542 to $2.7632 \mathrm{~kg} / \mathrm{m}^{3}$, and attenuation of $0.05 \mathrm{~dB} /$ wavelength. The third layer is a half-space with compressional velocity $5.4 \mathrm{~km} / \mathrm{s}$, density $2.7632 \mathrm{~kg} / \mathrm{m}^{3}$, and attenuation linearly increasing from 0.05 to $10 \mathrm{~dB} /$ wavelength over $100 \mathrm{~m}$, and a constant value of $10 \mathrm{~dB} /$ wavelength below. Bottom reflected energy strongly interferes with the energy contained in the first 10 modes only at short ranges. Therefore, for all numerically simulated wavefields at ranges of $1000 \mathrm{~km}$ and more, the bottom was assumed to be highly attenuating (with attenuation $10 \mathrm{~dB} /$ wavelength) to avoid numerical instabilities. The range step used in the RAM simulations was $79 \mathrm{~m}$ for T50 simulations and $50 \mathrm{~m}$ for all other simulations. The depth increment was $1 \mathrm{~m}$ in all simulations.

\section{Comparisons of measured and simulated acoustic wavefields}

Before looking at the modal statistics and making comparisons of data-based estimates with theoretical predictions, it is useful to compare data-based and numerically simulated wavefields in the time-depth domain. For convenience, we refer to transmissions made with a 800 or $500 \mathrm{~m}$ deep source as "axial" source transmissions, and all transmissions made with a $350 \mathrm{~m}$ deep source as "off-axial" source transmissions. The reason for this separation is qualitatively different behavior of energy redistribution among acoustic modes during propagation. An "axial" source initially excites all modes and the wavefield produced by such a source has a strong late finale near the sound channel axis (in the environments considered). However, an "off-axial" source initially does not excite energy in low order modes (or corresponding rays with small grazing angles). These energy levels are populated along the propagation path due to scattering, and corresponding wavefields do not have a strong late near-axial finale. The relative energy in the finale grows as the propagation range increases. One of the goals of the LOAPEX experiment was to study how the energy scatters into low mode numbers along the propagation path.

Figures 4 ("axial" source) and 5 ("off-axial" source) provide a comparison of the LOAPEX data and numerically simulated wavefields. One example is plotted for each transmission configuration (range, center frequency, source depth). The data pulses are coherently averaged as described in Sec. III B. The top two rows compare measured and simulated wavefield intensities as functions of depth and time. The bottom three rows compare three "cross sections" made at approximately 500,1000, and $1500 \mathrm{~m}$ depth. In general, the agreement between colored subplots (between measurements and simulations) is better at short ranges than at longer 
ranges. One reason for disagreement is the presence of variability in the background sound speed structure that is not accounted for in numerical simulations. However, some features present in these plots are worth emphasizing. First, the absolute levels of intensities at T500 and T1000 agree very well with values reported in Van Uffelen et al. (2009). (Note that in that paper the center frequency of acoustic source was $250 \mathrm{~Hz}$, suggesting that intensity levels should be slightly lower, which is exactly as observed). Second, the agreement of absolute levels of intensities of early arrivals at T50 is very good (within a fraction of a $\mathrm{dB}$ difference for axial source transmissions from T50). Third, at T250, arrival times of peaks mostly agree well, but the differences in absolute amplitudes are a few decibels. Fourth, careful examination of subplots corresponding to T50 transmissions suggests that the pulse shape generated by the source is not symmetric in time (it has longer trailing edge). Note that the time scale on each plot was chosen consistent with the scale chosen for the modal analysis presented in Sec. IV.

\section{MODAL PULSES IN LOAPEX}

This paper is concerned with the range evolution of modal pulses in LOAPEX. To address this topic, one needs to isolate broadband contributions to the wavefield from individual mode numbers and then study the statistics and range evolution of these distributions. In this paper, we focus on the first 10 modes resolved by the SVLA (first 2 modes for T2300 transmissions). In this section, mode filtering and computation of statistics of modal arrivals is discussed. Recall (e.g., Brown et al., 1996; Udovydchenkov and Brown, 2008) that to perform the mode filtering of transient wavefields one needs to: (1) Fourier transform measured time histories at each depth; (2) perform mode filtering at
T50
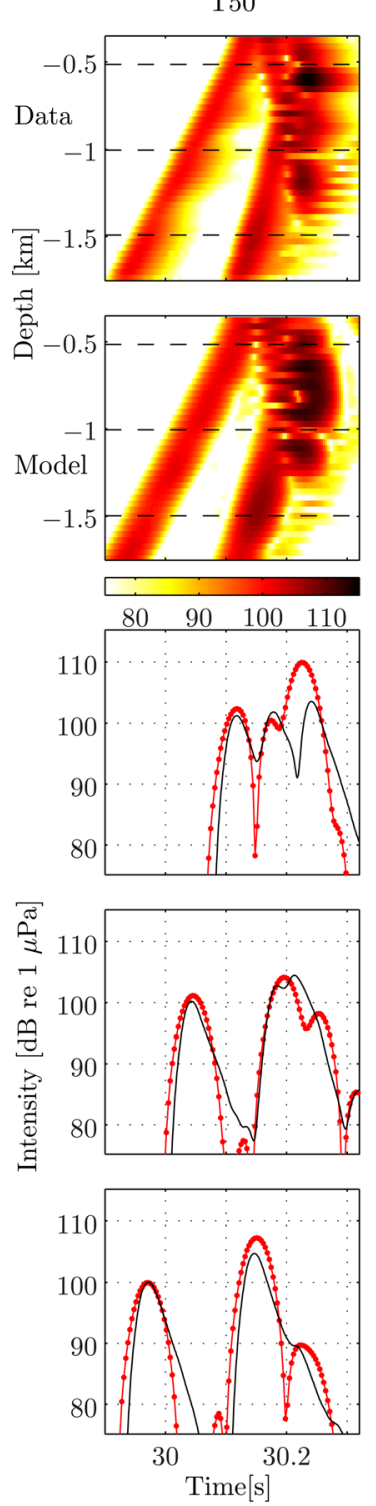

$\mathrm{T} 250$
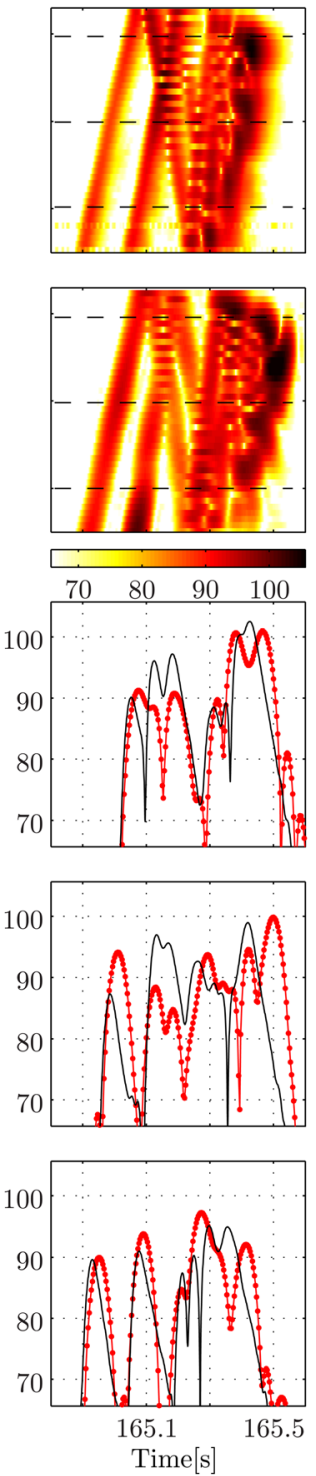

$\mathrm{T} 500$
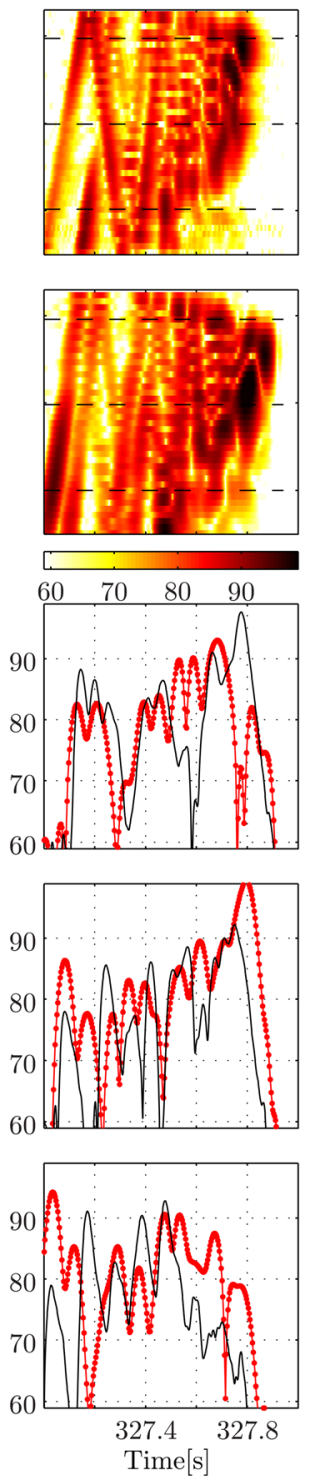

$\mathrm{T} 2300$
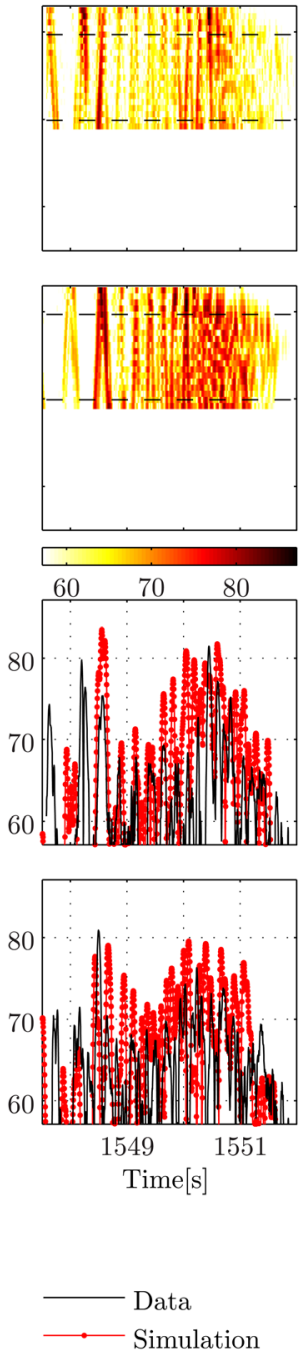

T3200
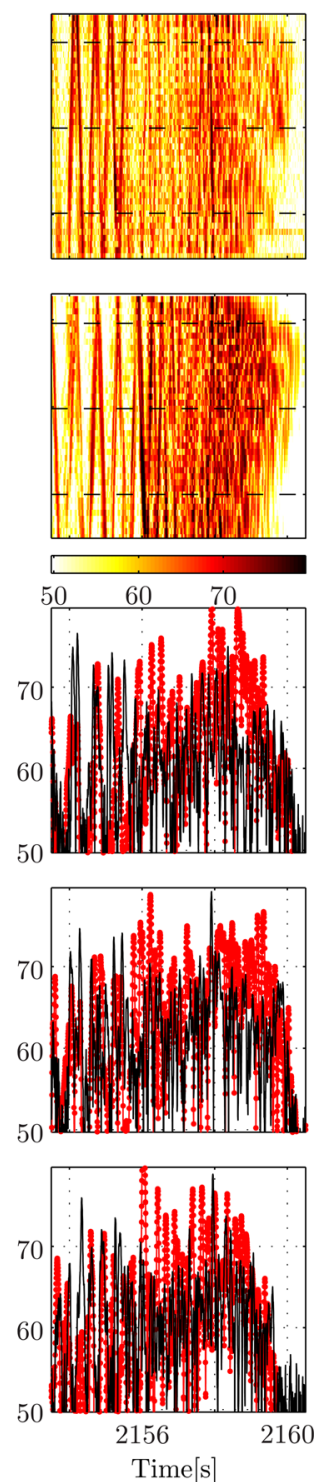

FIG. 4. (Color online). Absolute wavefield intensities as functions of absolute arrival time and depth are shown. Data and numerically simulated wavefields are compared. The top two rows show one example of the wavefield for each transmission configuration. The center frequency of the source is $75 \mathrm{~Hz}$ and the source depth is $800 \mathrm{~m}$ for T50, T250, and T500; and $68.2 \mathrm{~Hz}$ and $500 \mathrm{~m}$ for T2300 and T2300 transmissions. The bottom three rows compare measured and modeled wavefield intensities at 500, 1000, and $1500 \mathrm{~m}$ depth. 

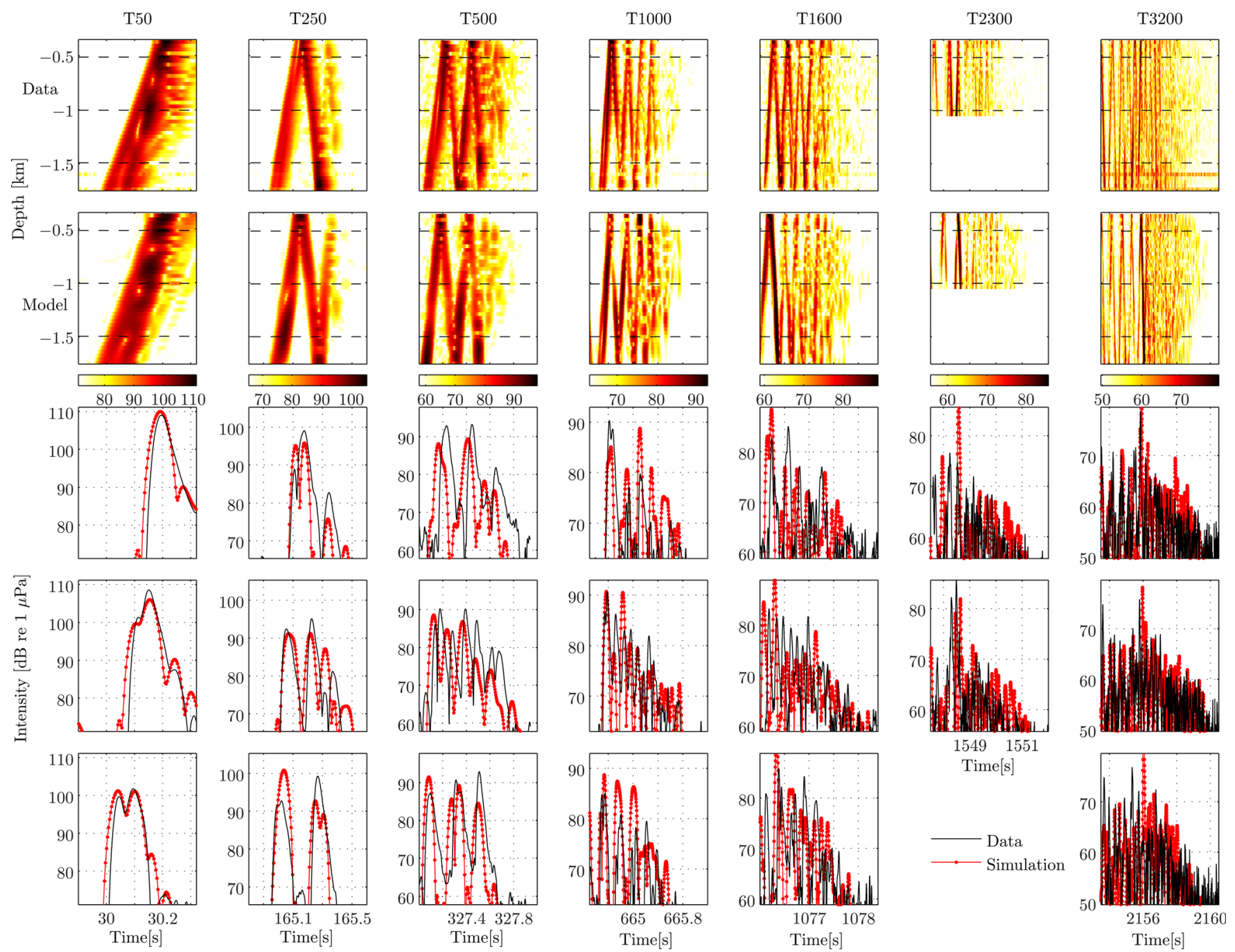

FIG. 5. (Color online). Same as Fig. 4, except that $68.2 \mathrm{~Hz}$ center frequency and $350 \mathrm{~m}$ source depth is used.

each frequency to isolate contributions to the wavefield from each mode number; and (3) inverse Fourier transform to construct transient contributions to the wavefield at each mode number (modal pulses). The mode filtering procedure relies on the orthogonality of normal modes and on the completeness of the set of eigenmodes. These functions are computed using the KRAKEN normal mode code.

\section{A. Mode processing}

There are several choices available for the mode filter including direct projection (DP, or sampled mode shapes), Moore-Penrose pseudo-inverse mode filter (PI), diagonally weighted mode filter (DW), or a maximum a posteriori mode filter (MAP) (Tindle et al., 1978; Buck et al., 1998; Wage et al., 2003; Wage et al., 2005; Golub and Van Loan, 1996). All of these filters are expected to perform well if properly configured. The mode filtering problem is naturally formulated as a linear inverse problem subject to an energy conservation constraint (Udovydchenkov et al., 2010); ideally solutions have small expected errors and good resolution (little cross-talk) and satisfy the energy conservation constraint.
In this paper, the Moore-Penrose PI mode filter was chosen because this filter effectively eliminates cross-talk between mode estimates and does not rely on any a priori information about the data or ad hoc parameters. The problem of solving the Helmholtz equation (1) for normal modes is generally formulated for a continuous medium, and solutions to this equation are continuous functions. These continuous functions are orthonormal and form a complete set. However, the DP mode filter relies on the orthogonality of sampled modal functions. Even though the number of hydrophones used in LOAPEX should be enough to resolve the first 10 modes, it is easy to come up with a configuration of the array covering the same depth aperture, for which DP filter will not work (for example, if spacing between hydrophones increases with depth). Because we are not using nominal hydrophone depths to construct wavefields but instead use the actual hydrophone depths for each transmission (filling the gaps in the mooring motion data with EOFs when necessary), the PI mode filter was chosen to avoid this potential problem. Under typical LOAPEX conditions, we expect that both DP and PI mode filters will perform well. Also note that PI mode filter satisfies the energy conservation condition. 
The SVLA was designed to resolve the first ten modes. The SVLA has 40 hydrophones with mean depths shown in the middle panel of Fig. 3. The modal "cross-talk" matrix shown in Fig. 3 confirms that these modes are well resolved. This matrix (Wage et al., 2003; Wage et al., 2005) was computed as $20 \times \log _{10}\left(\psi_{s}^{T} \psi_{s}\right)$, where $\psi_{s}$ is the matrix containing modal shapes at $75 \mathrm{~Hz}$ sampled at mean hydrophone depths during transmissions from T50. It is concluded that for all transmissions (except T2300, where data from lower part of the SVLA are not available), the first 10 modes $(m=0,1,2, \ldots, 9)$ are adequately resolved. The same plot constructed for the T2300 transmissions (upper half of the SVLA, not shown) suggests that for those transmissions modes $m=0$ and $m=1$ are resolved.

\section{B. Modal group time spreads}

To obtain reliable estimates of modal arrival statistics, one has to carefully consider the effects of variable SNR (which is generally low at long range) in the transmissions and presence of other signals (especially bottom reflections at short range). This subsection describes the algorithm that was used to compute modal statistics.

After coherently averaging modal pulses as described in Sec. III B, the acoustic wavefield was "time-gated" to discard bottom reflected signals (mostly at T50) and possibly high levels (often in the form of spikes) of ambient noise in low mode numbers at times where those arrivals can not be attributed to the transmitted signal (mostly at ranges of $1600 \mathrm{~km}$ or more). The reference sound speed $c_{0}$ was taken to be $1.478 \mathrm{~km} / \mathrm{s}$. The corresponding reference arrival time is $t_{0}=r / c_{0}$ (where $r$ is the transmission range). The time window around $t_{0}$ was chosen to be $\pm 0.5 \mathrm{sec}$ for T50 and $[-0.75 ; 0],[-1 ; 0],[-2 ; 0],[-3 ; 0],[-5 ; 0]$ and $[-8 ; 0] \mathrm{s}$ for $\mathrm{T} 250, \ldots, \mathrm{T} 3200$, respectively. Data outside of these windows were discarded. The resulting subsets were visually inspected to make sure that no signal had been discarded. Time windowing is necessary to eliminate any noise that can filter into low order modes at times significantly different from the signal arrival time and degrade estimates of pulse statistics. A noise floor level for every mode in every pulse was estimated using the $5 \mathrm{~s}$ long window that precedes the signal window. The maximum amplitude of the contribution from the noise was computed for every pulse and every mode number, and the data below these thresholds were discarded. Note that generally (especially at short transmission ranges) the noise floor estimated using this method is monotonically decreasing with increasing time between the two successive transmissions. This is due to reverberations present from the previously transmitted pulse that gradually decay until the next pulse arrives. It was noted that for axial source transmissions from T50 after these steps were performed the SNR was very high, sometimes as high as $50 \mathrm{~dB}$. However, because of the differences between the actual pulse shape (the spectrum of the signal radiated by the source is non-Gaussian and not symmetric) and the idealized pulse shape used in numerical simulations and theoretical estimates, the inclusion of the entire signal in the computation of modal statistics makes the comparison difficult. In addition, when the SNR is low, modal group time spreads may be largely underestimated when most of the pulse energy is below the noise floor and is discarded together with the noise. For this reason, when the SNR was higher than $20 \mathrm{~dB}$, the signal $20 \mathrm{~dB}$ or lower than the peak was zeroed. The first, second, and third moments were computed for every modal arrival for every pulse. Before converting estimated standard deviations into modal group time spreads consistent with conventions used in Eq. (6), the widths of pulses in the absence of noise were estimated. An idealized Gaussian pulse was constructed and standard deviations as a function of truncation level were computed (to mimic variable SNR). The data-based standard deviations were multiplied by the ratio of standard deviation of a Gaussian pulse with infinite SNR to the standard deviation of a Gaussian pulse with a given finite SNR. While this procedure, of course, can not recover the structure of the pulse below the noise floor, it eliminates the problem of time spreads being largely underestimated when SNR is small. Note that if this correction is not applied, the time spread of any isolated pulse approaches zero with SNR approaching zero (for example, the data pulse shown in Fig. 6 corresponding to the $m=1$ off-axial source transmission from T250). Skewnesses were computed as centered third moments of modal pulse amplitudes normalized by the standard deviation cubed for every mode number,

$$
\gamma(m)=\frac{\int(t-\bar{t}(m))^{3} q_{m}(t) d t}{\left(\int(t-\bar{t}(m))^{2} q_{m}(t) d t\right)^{3 / 2}},
$$

where $\bar{t}(m)=\int t q_{m}(t) d t$, and $q_{m}(t)$ denotes the normalized (unit area) modulus of the pressure time history (measured or simulated) corresponding to the mode number $m$.

Figure 6 shows examples of modal pulses for the first 10 modes for each transmission configuration. Mode amplitudes are plotted on a logarithmic scale with dynamic range of $20 \mathrm{~dB}$ on each subplot. To interpret this figure, recall that a Gaussian shaped pulse is parabolic on this scale. There are three sets of curves plotted. Black curves show modal pulses obtained from the LOAPEX data and red curves show simulated modal pulses. These two sets of curves are normalized together to the peak value in the data (except T2300, where peak values from the model were used) at each transmission range (so this figure allows comparison of the amplitude of mode $m$ in the data to the amplitude of mode $n$ in the simulation at a fixed range). The green curves correspond to theoretical predictions based on the model described in Sec. II C. The value of $B=1.0 \times 10^{-7} \mathrm{~s}^{2} / \mathrm{km}$ was used for all axial source transmissions and $B=1.5 \times 10^{-7} \mathrm{~s}^{2} / \mathrm{km}$ for all off-axial source transmissions. These values are consistent with previously reported estimates in (Udovydchenkov and Brown, 2008; Virovlyansky et al., 2007; Virovlyansky, 2006). Because these simple theoretical predictions cannot estimate absolute amplitudes of arrivals (only relative amplitudes are estimated), intensities shown by green lines are normalized separately (to the peak value at each transmission range).

Several important conclusions can be drawn from Fig. 6. First, at T50, it is evident (again) that the pulse shape 

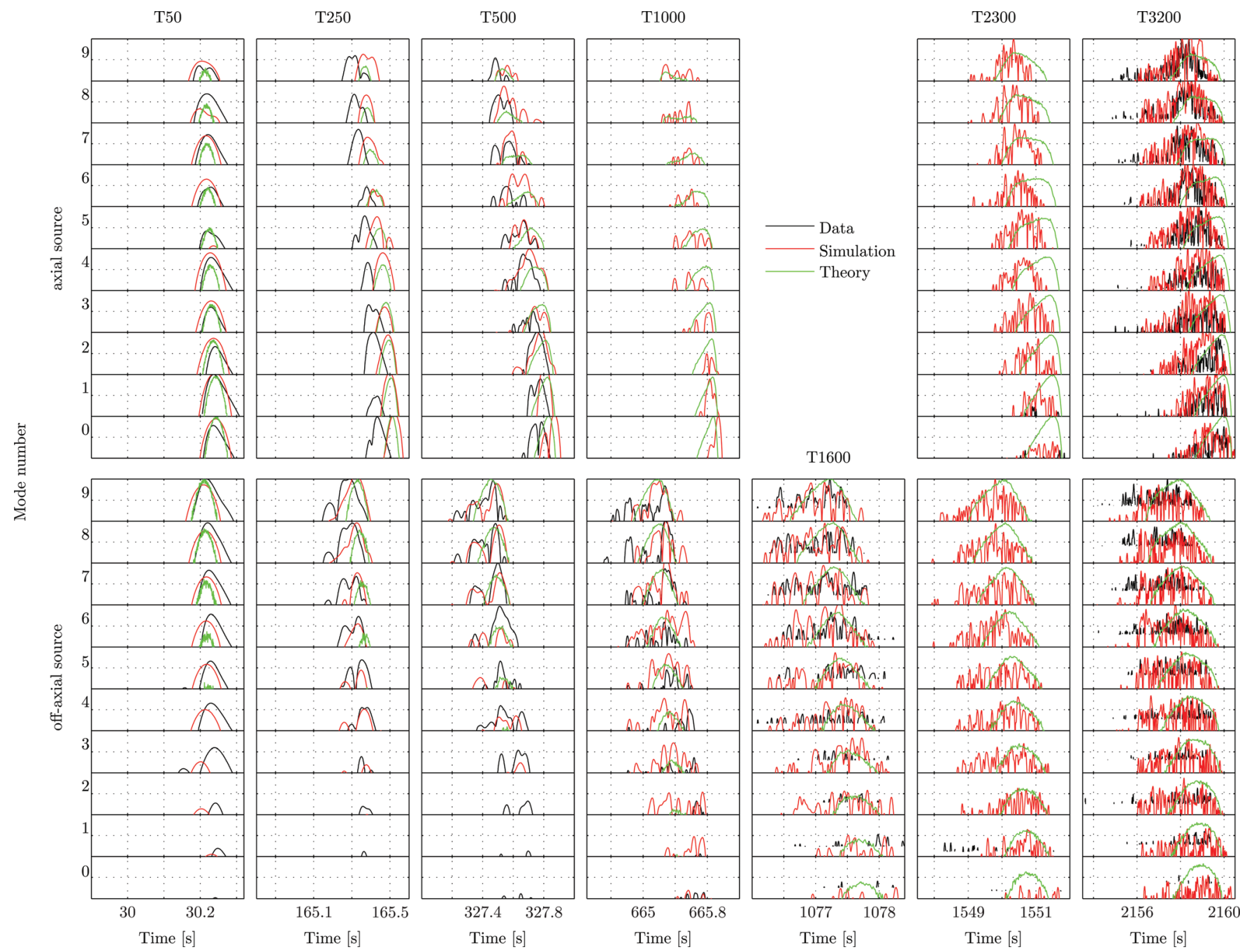

FIG. 6. Modal pulses in the LOAPEX. An example of one mode pulse for every transmission configuration is shown for the first 10 modes. Data are shown in black, the simulated pulses are shown in red, and theoretically predicted distributions of energy are shown in green. The dynamic range on every subplot is $20 \mathrm{~dB}$; the vertical grid spacing is $10 \mathrm{~dB}$.

used in the experiment is not symmetric in time because it has a long trailing edge. Second, one can easily see from these plots whether a particular group of modes is excited or not. As expected, an off-axial source does not excite low order modes at short ranges. However, the relative levels of excitation in measured and simulated wavefields agree well but do not agree with theoretical predictions. An explanation for this discrepancy was found in the presence of bottomreflected energy that interferes with main arrival. While bottom reflected signals do not contain any low mode number energy, the spacing between hydrophones in the SVLA is too coarse to discriminate the low mode energy from very high angle energy (bottom interacting energy with $m \gtrsim 100$ ). This hypothesis was confirmed by performing mode filtering of the data records past $30.3 \mathrm{~s}$ in transmissions from T50 (not shown) that contain only bottom-reflected arrivals. The third observation is the presence of persistent bias in arrival times for almost all modes for transmissions from T250. This observation suggests that $\mathrm{C} 250$ profile used for numerical simulations is not a good representation of the mean profile along the propagation path and is cold-biased. Comparison of T50 with C50 and T250 with C250 profiles in Fig. 3 reveals that both $\mathrm{C} 50$ (which is not used in simulations) and C250 are cold-biased with respect to the deep CTD profiles below the sound channel axis. This is the likely cause of the discrepancy in arrival times, but note that the deep CTD casts provide only two measurement points along the first $250 \mathrm{~km}$ and are clearly not sufficient to construct a realistic range-dependent environment. The fourth observation is that the simple theoretical model is able to correctly predict, qualitatively at least, the asymmetry (negative skewness) of modal arrivals at long ranges. Note that the theory predicts an envelope of arrival modal pulse, not an individual realization. The modal pulse, constructed from measurements and simulations that are shown in Fig. 6 are individual realizations of the wavefield and, of course, are not the same as theoretical prediction. At long ranges (for example the axial source transmissions from T3200), measurement-based distributions of energy clearly show pronounced long leading edge and abrupt trailing edge (negative skewness), especially for the lowest mode numbers, which are correctly (at least qualitatively) reproduced in both theoretical prediction and simulation. This figure also shows the SNR for each mode. While most of the modal pulses have sufficiently high 
SNR to allow modal statistics to be computed, in some instances (most of the T2300 transmissions, for example), low SNR makes the computation of modal statistics unreliable. Also low SNR and the lack of mesoscale variability in the numerical model results in larger disagreement of arrival amplitudes of the evanescent axial energy (corresponding to low mode numbers) for transmissions made from T2300 and T3200 stations with the off-axial source.

\section{RESULTS}

In this section, we present the results of mode processing the LOAPEX measurements, and compare measurement-based statistics of modal group arrivals with both full wave simulations and theoretical predictions. The entire LOAPEX data set has been analyzed; the number of transmissions used in our analysis is given in Table I. Full wave numerical simulations using RAM were done once (with a single realization of internal-wave-induced perturbation) for each transmission configuration. (Unfortunately, computing an ensemble of wavefield realizations for each transmission configuration would be computationally prohibitive.) Theoretical estimates of modal group time spreads were based on the theory-based numerical model described in Sec. II C.

\section{A. Statistics of modal pulses in LOAPEX}

In this subsection, the statistics of modal group time spreads are analyzed. The dependence of modal group time spreads on both range and mode number is considered. Figure 7 shows modal group time spread statistics as a function of range for each mode number. In the absence of scattering, modal pulses grow, approximately linearly, with increasing range due to dispersion. It is convenient to remove the linear trend in modal group time spreads by considering spreads in group slowness [recall Eq. (4)], rather than time spreads. Group slowness spreads $\Delta S_{g}$ are plotted in Figs. 7 and 8 . Note that as $r \rightarrow 0, \Delta S_{g} \rightarrow \infty$ because of the constant reciprocal bandwidth contribution term (7).

Three different sets of group slowness spreads are plotted on each subplot of Fig. 7. These are measurement-based estimates (sample means over the transmissions listed in Table I), one measure based on full wave simulations, and the theoretical predictions. Two different measures of the goodness of measurement-based estimates are also plotted with vertical error bars. The outer (wider) error bars represent the $90 \%$ confidence interval for the individual

TABLE I. Overview of LOAPEX transmissions analyzed in this paper. The number of transmissions used in the analysis for each transmission type and at each range is given. Each transmission corresponds to one 1023 digit $\mathrm{m}$ sequence. The complete transmission schedule can be found in Mercer et al. (2005).

\begin{tabular}{lccccccc}
\hline \hline & T50 & T250 & T500 & T1000 & T1600 & T2300 & T3200 \\
\hline SVLA (km) & 44.714 & 244.7 & 484.7 & 984.7 & 1594.7 & 2294.7 & 3194.7 \\
75 Hz 800 m & 330 & 363 & 462 & - & - & - & - \\
$68.2 \mathrm{~Hz} \mathrm{350m}$ & 240 & 330 & 200 & 300 & 810 & 504 & 540 \\
$68.2 \mathrm{~Hz} 500 \mathrm{~m}$ & - & - & - & - & - & 540 & 612 \\
\hline \hline
\end{tabular}

realization (i.e., the interval within which a single estimate of modal group time spread would fall with $90 \%$ probability). Comparing these intervals with theoretical predictions is misleading because the theory does not predict an individual wavefield realization, but provides the mean modal group time spread. The second plotted measure of goodness of these estimates is the confidence interval for the mean (Bendat and Piersol, 2010, pp. 88-90), which shows the interval within which the mean of the estimate would fall with $90 \%$ probability if the entire experiment was repeated. The confidence interval of the mean is plotted with the inner error bars on top of each measurement-based estimate.

Many factors influence the agreement among data, simulations, and theory. For axial source transmissions (top two rows of Fig. 7) from T50, the data usually show larger spread than predicted by the theory. There are two reasons for this: (1) the shape of the source spectrum and (2) bottom reflections. Modal group time spread estimates at this range are strongly controlled by the reciprocal bandwidth contribution (7). Estimated values of modal group time spreads at this range suggest that the true spectrum of the source used in LOAPEX is narrower than the spectrum of the idealized reference signal used in the processing. Based on earlier tests of the source, our initial estimate of the source bandwidth (full width at half amplitude) was approximately $20 \mathrm{~Hz}$. However, the modal group time spreads from T50 suggest that the true width should be around $16 \mathrm{~Hz}$. Our full wave simulations were done with $\Delta f=18.75 \mathrm{~Hz}$ with $75 \mathrm{~Hz}$ center frequency ( $\Delta f=17.05 \mathrm{~Hz}$ with $68.2 \mathrm{~Hz}$ center frequency), which is intermediate between these estimates. The second reason for the large data-based estimates is the presence of bottom-interacting energy. As discussed previously, this energy is not completely filtered out by the array and results in time spreads being larger than theoretically predicted. Additional complications arise when a mode (or a group of modes) is not strongly excited. This happens when the acoustic source depth nearly coincides with the node of a particular mode. In the axial source transmissions from T50 that are shown in Fig. 7, modes $m=5,6$, and 9 are weakly excited (note that in the corresponding full wave simulations modes $m=5,8$ and 9 are weakly excited). For these modes, estimation of spreads is difficult and error bars are large. Also, error bars are always large (sometimes outside of the subplot) for modes that are not excited by the off-axial source. In light of this discussion, the data that are the easiest to analyze and show the best agreement with theory correspond to T250 and T500 transmissions with an axial source.

Modal group time spreads grow monotonically with range, but the spread in group slowness is predicted to increase at both short and long ranges. Using Eqs. (6)-(9), one can estimate the range at which the spread $\Delta S_{g}$ is expected to be minimal. This range is equal to

$$
r_{m s}=\frac{1}{\pi}\left(\frac{3}{8 B}\right)^{1 / 3}\left(\frac{R}{|\beta| \Delta f}\right)^{2 / 3} .
$$

To obtain an approximate quantitative estimate of $r_{m s}$ we take $B=1.0 \times 10^{-7} \mathrm{~s}^{2} / \mathrm{km}, R \approx 50 \mathrm{~km}, \Delta f=18.75 \mathrm{~Hz}$, $|\beta| \approx 0.2$, giving $r_{m s} \approx 280 \mathrm{~km}$. The upper part of the Fig. 7 

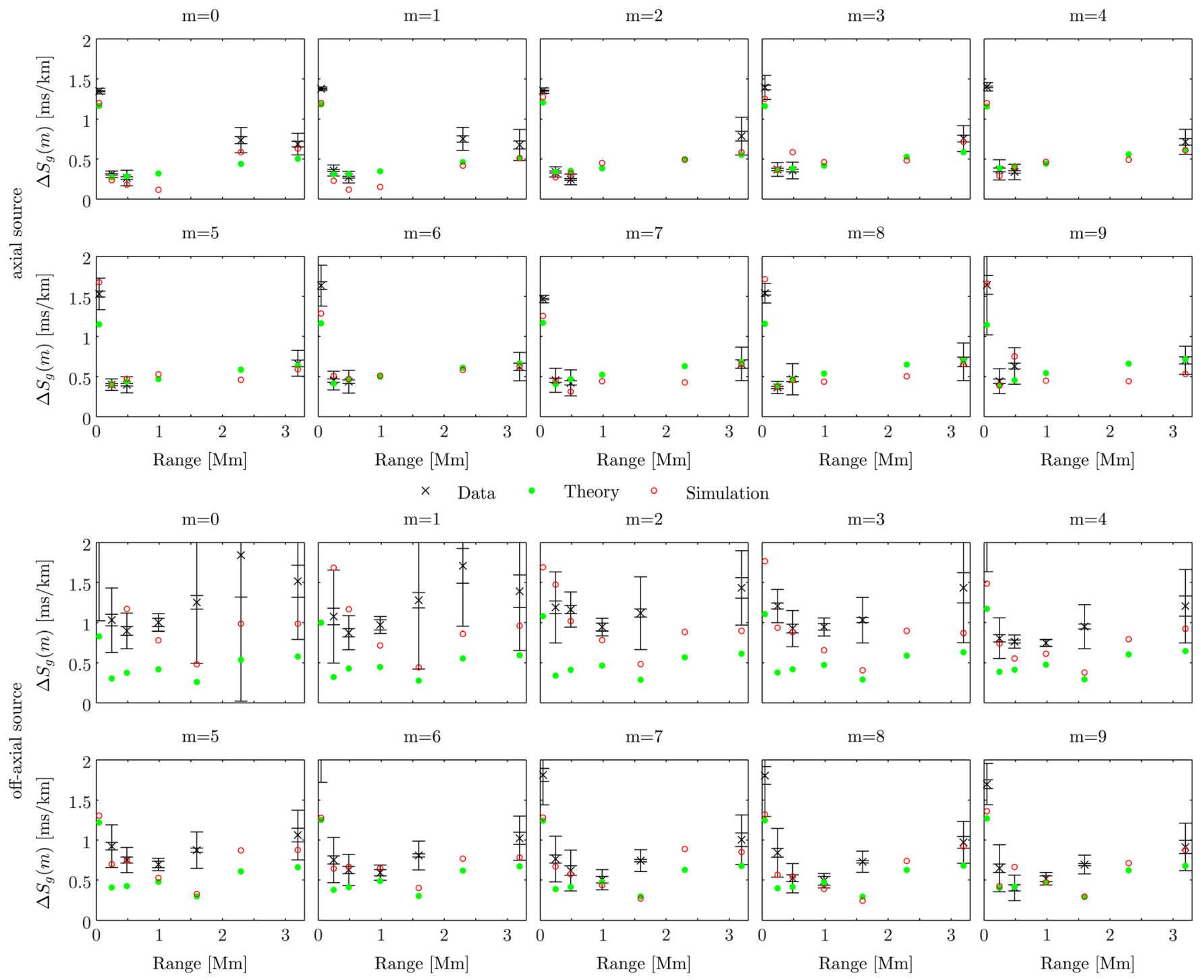

FIG. 7. (Color online). Modal group slowness spreads as functions of range for the first ten modes. Measurement-based estimates are shown with "x"-symbols together with 2 sets of error bars as described in the text. Full-wave-simulation- based slowness spreads are shown with open circles. Theory-based estimates of slowness spreads are shown using filled circles. The top two rows show results for axial source transmissions; the bottom two rows show results for offaxial source transmissions.

(corresponding to the axial source transmissions) suggests that group slowness spread is smallest at ranges between 250 and $1000 \mathrm{~km}$. Note that this minimum is very broad, its position is mode number dependent, and Eq. (9) overestimates near-axial spreads by a mode-number-dependent factor that is close to 2. The latter observation leads us to expect that the estimate $r_{m s} \approx 280 \mathrm{~km}$ is slightly low. Because of all of the factors that we have described, agreement with LOAPEX observations is about as good as can be expected.

Figure 8 shows modal group time spreads and skewnesses as functions of mode number at fixed ranges. For the T50 axial source transmission, predicted spreads are consistently smaller than the data-based estimates, suggesting that the true effective frequency bandwidth of the source was slightly less than $18.75 \mathrm{~Hz}$. Note that the "outliers" of the simulation-based estimates correspond to weakly excited modes. The agreement among theory, simulations, and measurements is very good for axial source transmissions at T250 and T500. For off axial source transmissions, the general trend is that the larger the mode number, the better the agreement. This is largely due to the difficulty of estimating time spreads for modes that are weakly excited.

The two bottom rows of Fig. 8 compare data-based, simulated, and theoretically predicted skewnesses as functions of mode number. Skewness error bars were computed using the same methods that were used to compute time spreads error bars. As expected, for the axial source transmissions from T50, the data-based skewnesses are positive (because of long trailing edge in the shape of the pulse) and simulation- and theory-based estimates are close to zero. The agreement for axial source transmissions from T250 and T500 is very good. At T3200, the agreement between simulations and data is good, but comparison with theory is mostly qualitative; all skewnesses are predicted to be negative. The agreement for off-axial source transmissions is generally very good for transmissions made from T1600 to T3200 stations. It is also good between the data and full wave numerical simulations at shorter ranges with the 


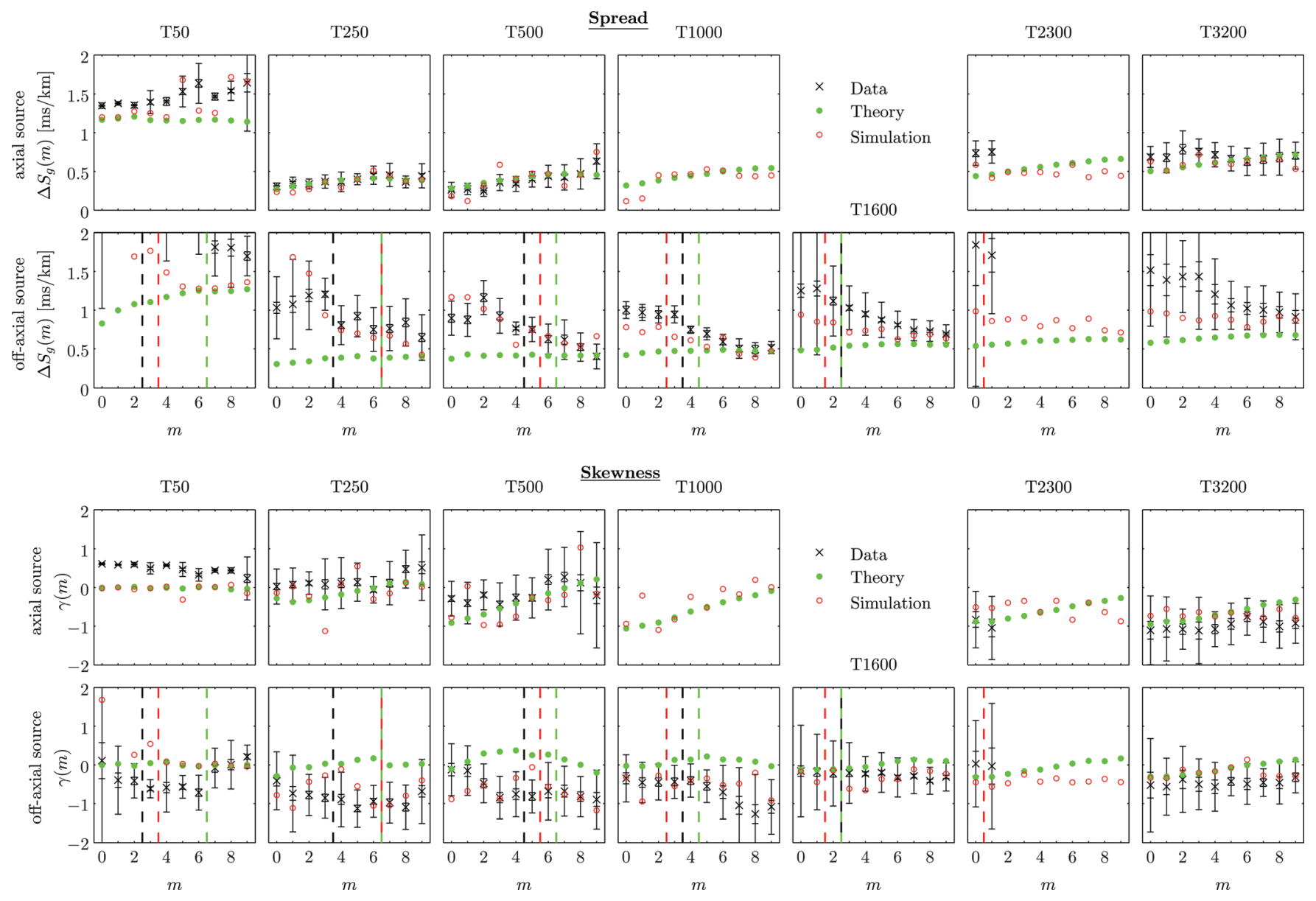

FIG. 8. (Top two rows) Modal group times spreads as functions of mode number for each transmission configuration. Annotation is the same as in Fig. 7. (Bottom two rows) Measured, simulation-based, and theoretically predicted skewnesses as functions of mode number. Vertical dashed color lines on subplots corresponding to the off-axial source transmissions separate "excited" from "not excited" modes as defined in the text.

caveats mentioned in the preceding text relating to weakly excited modes. The simple numerical model tends to overestimate the skewnesses for transmissions made from intermediate ranges T250-T1000.

\section{B. Scattering of acoustic energy into low mode numbers}

An important issue that was investigated is the rangedependence along the propagation path of acoustic energy of low-order modes that are not excited by an off-axial source. To study how the energy redistributes among low order modes due to scattering, first we need to identify an appropriate criterion that distinguishes an "excited" from a "not excited" mode number. One might, for example, compute the total energy (integrated over time) contained in every mode pulse and argue that if this energy is less than a certain threshold (half of the total energy in mode $m=10$, say), then the mode is not excited. This criterion has the shortcoming of not allowing one to distinguish between two scenarios: (a) a strongly excited mode, but with short duration vs (b) a weakly excited mode with long duration. After several attempts to quantify this effect, we decided to use instead a " $10 \mathrm{~dB}$ threshold" criterion. According to this criterion, we call a mode "excited" if the peak amplitude of that mode is less than $10 \mathrm{~dB}$ below the peak of any mode (among first 10) in that transmission. This criterion proved to be robust. One should not, however, be mislead by the term not excited. A mode amplitude can be several decibels above the noise floor, but for the analysis presented in this section, we call it not excited if the peak amplitude is more than $10 \mathrm{~dB}$ below the peak of any mode in that transmission. Of course, when analyzing many transmissions of the same configuration, a particular mode can be excited in one realization and not excited in another. So, we prescribe the value +1 if a mode is excited and -1 if it is not in a particular realization. If the mean value of the total series for a mode is nonnegative, then we identify this mode as excited; otherwise it is not excited. Note also that we only use this analysis for the offaxial source transmissions.

The number of not excited modes for data, simulations, and theoretical predictions as a function of range is given in the Table II and plotted using color-coded vertical lines in Fig. 8 (black for the LOAPEX data, red for RAM, and green for theory). The mode numbers to the right from each vertical line are excited, and the ones to the left are not excited. Only at ranges of 50 and $250 \mathrm{~km}$ do data and simulations deviate significantly from theoretical predictions. At both ranges, the cause is bottom-reflected energy. The theory does not account for bottom-reflected energy, but both simulations and data show strong bottom-reflected arrivals at T50. Recall that this energy is, in fact, not low-mode number 
TABLE II. Summary of "not excited" number of modes (defined in the text) observed in the data and estimated using full wave numerical simulation and theory as a function of range for the off-axial source transmissions.

\begin{tabular}{lccccccc}
\hline \hline & T50 & T250 & T500 & T1000 & T1600 & T2300 & T3200 \\
\hline Data & 3 & 4 & 5 & 4 & 3 & 0 & 0 \\
Model & 4 & 7 & 6 & 3 & 2 & 1 & 0 \\
Theory & 7 & 7 & 7 & 5 & 3 & 0 & 0 \\
\hline \hline
\end{tabular}

energy, but appears as such because of coarse spacing between hydrophones. At ranges longer that $250 \mathrm{~km}$, when comparing data, simulations, and theory, the dividing line between excited and not excited modes differs by at most one mode number. We consider this agreement to be good.

\section{SUMMARY}

In this paper, a modal analysis of the data recorded on the SVLA during the LOAPEX experiment with focus on the first 10 propagating modes was presented. The basic theory of modal group time spreads was reviewed, and a necessary extension for proper treatment of low order modes was described. A simplified numerical model based on the theory presented was developed. The entire SVLA-based LOAPEX data set was processed and modal pulse statistics were computed. Data-based estimates of modal group time spreads and skewnesses were compared to theoretical predictions and estimates from full wave numerical simulations. Overall agreement among the theory, numerical simulations, and data is good. In instances with poor agreement, the causes were identified and explained.

Complexities associated with low mode number scattering were described in detail. It was shown that in a typical mid-latitude ocean environment at ranges of $250 \mathrm{~km}$ or more and in the $50-100 \mathrm{~Hz}$ band that the correct low-m modal group time spreads estimates are approximately one half as large as the large-m theory predicts for the lowest mode numbers. The theoretical formulation used in this paper does not lead to a simple analytical expression for the scatteringinduced contribution to modal group time spreads. However, the underlying theoretical framework led to the development of a simple numerical model based on a random walk process with a reflecting boundary. This numerical model is capable of predicting modal group time spreads for receptions that do not have strong interference with bottom reflected energy (this model does not account for bottom-reflected energy). This model is also capable of predicting skewnesses of arrival modal pulses, but the agreement with data is mostly qualitative (positive, near-zero, or negative).

Agreement between measurement-based estimates of modal group time spreads and skewnesses with estimates based on full wave simulations and theory was best for the T250 and T500 transmissions. At shorter range (T50 transmissions), receptions were complicated by the presence of bottom reflected energy. These arrivals are not filtered out by the SVLA and cause the estimates of modal group time spreads to be too large. Realistic bottom properties are required to achieve good agreement in the excited mode number cutoff between data-based estimates and RAM-based estimates at this short range. It was confirmed that RAM-based estimates agree well with theoretical predictions at short ranges if a dense simulated array of hydrophones is used and bottomreflected energy is properly filtered out. At longer ranges (T1000, T1600, T2300, and T3200), low SNR and background range dependence led to some discrepancies in data/ simulation/theory comparisons. Coherent averaging was used to improve SNR for all transmissions. Noise levels were estimated for each transmission (after coherent averaging) and each mode number. However, we did not conduct an extensive study of signal coherence and did not attempt to find the optimal averaging time or estimate the signal decorrelation time. Agreement between modal group time spreads is generally good for all axial source transmissions for strongly excited mode numbers. For off-axial source transmissions, the general trend is that the larger the mode number, the better the agreement. It is noteworthy that all skewness estimates correctly predict negatively skewed distributions at long ranges (transmissions from T2300 and T3200).

Unfortunately, because the SVLA only allows the resolution of the first ten modes, it was not possible to fully test the predicted dependence of modal group time spreads on $\beta$. A shortcoming of our analysis is linked to the assumption in the theoretical formulation that was applied that the background sound speed structure is range-independent. Even in the LOAPEX environment where mesoscale variability was weak, this assumption led to some ambiguities and errors. For example, a persistent travel time bias, related to a bias in the assumed background sound speed profile, was seen in the T250 receptions.

In other deep ocean environments, mesoscale variability is much more energetic than in the LOAPEX environment. For many purposes, it is natural to treat this mesoscale structure as deterministic, i.e., as part of the background structure to which a stochastic perturbation, generally associated with internal waves, is superimposed. A critically important element of any theory of sound scattering and mode coupling in such environments is accounting for this background rangedependent sound speed structure. This represents a significant challenge that needs to be addressed.

\section{ACKNOWLEDGMENTS}

We thank Lora Van Uffelen for analyzing and providing access to the LOAPEX CTD data. We thank Irina Rypina, Ralph Stephen, Ying-Tsong Lin, Kathleen Wage, Tarun Chandrayadula, Brian Dushaw, and Frank Henyey for the benefit of many discussions related to this paper. We also thank the reviewers who provided valuable feedback on the manuscript. This work was supported by the Office of Naval Research, Code 322, Grant Nos. N00014-08-1-0195, N00014-06-1-0245, and N0014-11-1-0194.

\section{APPENDIX}

In this appendix an expression for $P\left(I_{1}, I_{2}, r_{1}, r_{2} \mid I_{0}\right)$ used in Eq. (13) is derived. The joint probability density function 
$P\left(I_{1}, I_{2}, r_{1}, r_{2} \mid I_{0}\right)$ describes a random walk process $I(r)$ with the initial condition $I(r=0)=I_{0}$. At ranges $r_{1}$ and $r_{2}$ (assuming $\left.r_{2} \geq r_{1}\right)$, it takes the values $I \quad\left(r_{1}\right)=I_{1}$ and $I\left(r_{2}\right)=I_{2}$. Because $I(r)$ is a Markov process,

$$
P\left(I_{1}, I_{2}, r_{1}, r_{2} \mid I_{0}\right)=P\left(I_{2}, r_{2}-r_{1} \mid I_{1}\right) P\left(I_{1}, r_{1} \mid I_{0}\right) .
$$

Substituting Eq. (15) into Eq. (A1) yields

$$
\begin{aligned}
P\left(I_{1}, I_{2}, r_{1}, r_{2}, \mid I_{0}\right)= & \frac{1}{2 \pi B \sqrt{r_{1}\left(r_{2}-r_{1}\right)}}\left[e^{-\frac{\left(I_{1}-I_{0}\right)^{2}}{2 B r_{1}}}+e^{-\frac{\left(I_{1}+I_{0}\right)^{2}}{2 B r_{1}}}\right] \\
& \times\left[e^{-\frac{\left(I_{2}-I_{1}\right)^{2}}{2 B\left(r_{2}-r_{1}\right)}}+e^{-\frac{\left(I_{2}+I_{1}\right)^{2}}{2 B\left(r_{2}-r_{1}\right)}}\right],
\end{aligned}
$$

which is substituted into Eq. (13) for numerical evaluation and construction of the right panel of Fig. 2. The crosscorrelation of the two processes $I_{1}\left(r_{1}\right)$ and $I_{2}\left(r_{2}\right)$ is

$$
\begin{aligned}
C\left(r_{1}, r_{2}\right) & =\left\langle I_{1}\left(r_{1}\right) I_{2}\left(r_{2}\right)\right\rangle \\
& =\int_{0}^{\infty} d I_{1}\left(I_{1}-\bar{I}\left(r_{1}\right)\right) \int_{0}^{\infty} d I_{2}\left(I_{2}-\bar{I}\left(r_{2}\right)\right) P\left(I_{1}, I_{2}, r_{1}, r_{2} \mid I_{0}\right),
\end{aligned}
$$

and the variance of the integrated random walk process is

$$
\sigma_{t}^{2}=\int_{0}^{r} \int_{0}^{r} C\left(r_{1}, r_{2}\right) d r_{1} d r_{2}
$$

Andrew, R. K., Zarnetske, M. R., Howe, B. M., and Mercer, J. A. (2010). "Ship-suspended acoustical transmitter position estimation and motion compensation," IEEE J. Ocean. Eng. 35, 797-810.

Beichelt, F. (2006). Stochastic Processes in Science, Engineering and Finance (Chapman and Hall/CRC, Taylor and Francis Group, Boca Raton, FL), Chap.7, pp. 357, 358, 387-389.

Bendat, J. S., and Piersol, A. G. (2010). Random Data. Analysis and Measurement Procedures (Wiley and Sons, Hoboken, NJ), Chap. 4.4, pp. 88-90.

Beron-Vera, F. J., Brown, M. G., Colosi, J. A., Tomsovic, S., Virovlyansky, A. L., Wolfson, M. A., and Zaslavsky, G. M. (2003). "Ray dynamics in a long-range acoustic propagation experiment," J. Acoust. Soc. Am. 114, 1226-1242.

Brown, M. G., Viechnicki, J., and Tappert, F. D. (1996). "On the measurement of modal group time delays in the deep ocean," J. Acoust. Soc. Am. 100, 2093-2102.

Buck, J. R., Preisig, J. C., and Wage, K. E. (1998). "A unified framework for mode filtering and the maximum a posteriori mode filter," J. Acoust. Soc. Am. 103, 1813-1824.

Chandrayadula, T. K. (2009). "Mode tomography using signals from the long range ocean acoustic propagation experiment (LOAPEX)," Ph.D. thesis, George Mason University, Fairfax, VA.

Chandrayadula, T. K., and Wage, K. E. (2008). "Interpolation methods for vertical linear array element localization," in Proceedings of the 2008 IEEE/MTS Oceans Conference, Quebec City, Canada, p. 1-5.

Collins, M. (1993). "A split-step Padé solution for the parabolic equation method," J. Acoust. Soc. Am. 93, 1736-1742.

Collins, M. D., and Westwood, E. K. (1991). "A higher-order energy-conserving parabolic equation for range-dependent ocean depth, sound speed and density," J. Acoust. Soc. Am. 89, 1068-1075.

Colosi, J. A., and Brown, M. G. (1998). "Efficient numerical simulation of stochastic internal- wave-induced sound speed perturbation fields," J. Acoust. Soc. Am. 103, 2232-2235.

Colosi, J. A., Scheer, E. K., Flatté, S. M., Cornuelle, B. D., Dzieciuch, M. A., Munk, W. H., Worcester, P. F., Howe, B. M., Mercer, J. A., Spindel, R. C., Metzger, K., Birdsall, T. G., and Baggeroer, A. B. (1999). "Comparison of measured and predicted acoustic fluctuations for a 3250-km propagation experiment in the eastern North Pacific Ocean," J. Acoust. Soc. Am. 105, 3202-3218.

Colosi, J. A., Tappert, F. D., and Dzieciuch, M. A. (2001). "Further analysis of intensity fluctuations from a $3252-\mathrm{km}$ acoustic propagation experiment in the eastern North Pacific Ocean," J. Acoust. Soc. Am. 110, 163-169.

Cornuelle, B. D., Worcester, P. F., Hildebrand, J. A., Hodgkiss, W. S., Jr., Duda, T. F., Boyd, J., Howe, B. M., Mercer, J. A., and Spindel, R. C. (1993). "Ocean acoustic tomography at 1000-km range using wavefronts measured with a large-aperture vertical array," J. Geophys. Res. 98, 16365-16377.

Del Grosso, V. A. (1974). "New equation for the speed of sound in natural waters (with comparisons to other equations)," J. Acoust. Soc. Am. 56, 1084-1091.

Duda, T. F., Flatté, S. M., Colosi, J. A., Cornuelle, B. D., Hildebrand, J. A., Hodgkiss, W. S., Jr., Worcester, P. F., Howe, B. M., Mercer, J. A., and Spindel, R. C. (1992). "Measured wave-front fluctuations in 1000-km pulse propagation in the Pacific ocean," J. Acoust. Soc. Am. 92, 939-955.

Dushaw, B. D., and Colosi, J. A. (1998). "Ray tracing for ocean acoustic tomography," Technical Report APL-UW TM 3-98, Applied Physics Laboratory, University of Washington, Seattle, WA.

Emery,W. J., and Thomson, R. E. (2004). Data Analysis Methods in Physical Oceanography (Elsevier, San Diego, CA), Chap. 4, pp. 319-343.

Flatté, S., Dashen, R., Munk, W., Watson, K., and Zachariasen, F. (1979). Sound Transmission Through a Fluctuating Ocean, Mechanics and Applied Mathematics (Cambridge University Press, New York).

Golub, G., and Van Loan, C. F. (1996). Matrix Computations (The Johns Hopkins University Press, Baltimore, MD).

Jensen, F. B., Kuperman, W. A., Porter, M. B., and Schmidt, H. (2000). Computational Ocean Acoustics (Springer-Verlag, New York).

Lin, Y.-T., Newhall, A. E., Duda, T. F., Lermusiaux, P. F. J., and Haley, P. (2010). "Merging multiple-partial-depth data time series using objective empirical orthogonal function fitting," IEEE J. Ocean. Eng. 35, 710-721.

Makarov, D., Prants, S., Virovlyansky, A. L., and Zaslavsky, G. (2010). Ray and Wave Chaos in Ocean Acoustics (World Scientific, Hackensack, NJ).

Mercer, J. A., Andrew, R. K., Howe, B. M., and Colosi, J. A. (2005). "Cruise report: Long-range ocean acoustic propagation experiment (LOAPEX)," Technical Report APL-UW TR0501, Applied Physics Laboratory, University of Washington, Seattle, WA.

Mercer, J. A., Colosi, J. A., Howe, B. M., Dzieciuch, M. A., Stephen, R., and Worcester, P. F. (2009). "LOAPEX: The Long-Range Ocean Acoustic Propagation Experiment,” IEEE J. Ocean. Eng. 34, 1-11.

Munk, W., Worcester, P., and Wunsch, C. (1995). Ocean Acoustic Tomography (Cambridge University Press, New York).

Porter, M. B. (1991). "The KRAKEN normal mode program. rep. sm-245," Technical Report, SACLANT Undersea Research Centre, La Spezia, Italy. Sea-Bird Electronics, Inc. (2003a). http://www.seabird.com/products/spec_ sheets/37smdata.htm (Last viewed on 03/01/2012).

Sea-Bird Electronics, Inc. (2003b). http://www.seabird.com/products/spec_ sheets/39data.htm (Last viewed on 03/01/2012).

Smith, W. H. F., and Sandwell, D. T. (1997). "Global seafloor topography from satellite altimetry and ship depth soundings," Science 277, 1956-1962.

Stephen, R. A., Bolmer, S. T., Dzieciuch, M. A., Worcester, P. F., Andrew, R. K., Buck, L. J., Mercer, J. A., Colosi, J. A., and Howe, B. M. (2009). "Deep seafloor arrivals: An unexplained set of arrivals in long-range ocean acoustic propagation,” J. Acoust. Soc. Am. 126, 599-606.

Tindle, C. T., Guthrie, K. M., Bold, G. E. J., Johns, M. D., Jones, D., Dixon, K. O., and Birdsall, T. G. (1978). "Measurements of the frequency dependence of normal modes," J. Acoust. Soc. Am. 64, 1178-1185.

Udovydchenkov, I. A., and Brown, M. G. (2008). "Modal group time spreads in weakly range-dependent deep ocean environments," J. Acoust. Soc. Am. 123, 41-50.

Udovydchenkov, I. A., Rypina, I. I., and Brown, M. G. (2010). "Mode filters and energy conservation,” J. Acoust. Soc. Am. 127, EL185-EL191.

Van Uffelen, L. J., Worcester, P. F., Dzieciuch, M. A., and Rudnick, D. L. (2009). "The vertical structure of shadow-zone arrivals at long range in the ocean," J. Acoust. Soc. Am. 125, 3569-3588.

Van Uffelen, L. J., Worcester, P. F., Dzieciuch, M. A., Rudnick, D. L., and Colosi, J. A. (2010). "Effects of upper ocean sound-speed structure on deep acoustic shadow-zone arrivals at 500- and 1000-km range," J. Acoust. Soc. Am. 127, 2169-2181.

Virovlyansky, A. L. (2006). Ray Theory of Long-Range Sound Propagation in the Ocean (in Russian) (Institute of Applied Physics, Nizhny Novgorod, Russia). 
Virovlyansky, A. L., Kazarova, A. Y., and Lyubavin, L. Y. (2006). "Modal structure of the field under conditions of wave chaos," in Ocean Acoustics. Proceedings of the 11-th L. M. Brekhovskikh's Conference. Moscow, Russia, CEOS (in Russian), pp. 40-43.

Virovlyansky, A. L., Kazarova, A. Y., and Lyubavin, L. Y. (2007). "Statistical description of chaotic rays in a deep water acoustic waveguide," J. Acoust. Soc. Am. 121, 2542-2552.

Virovlyansky, A. L., Kazarova, A. Y., and Lyubavin, L. Y. (2009). "Raybased description of normal modes in a deep ocean acoustic waveguide," J. Acoust. Soc. Am. 125, 1362-1373.

Virovlyansky, A. L., Kazarova, A. Y., and Lyubavin, L. Y. (2011). "Raybased description of shadow zone arrivals," J. Acoust. Soc. Am. 129, 2851-2862.

Wage, K. E., Baggeroer, A. B., and Preisig, J. C. (2003). "Modal analysis of broadband acoustic receptions at $3515-\mathrm{km}$ range in the North
Pacific using short-time Fourier techniques,” J. Acoust. Soc. Am. 113, 801-817.

Wage, K. E., Dzieciuch, M. A., Worcester, P. F., Howe, B. M., and Mercer, J. A. (2005). "Mode coherence at megameter ranges in the North Pacific Ocean," J. Acoust. Soc. Am. 117, 1565-1581.

Worcester, P. F., Cornuelle, B. D., Dzieciuch, M. A., Munk, W. H., Howe, B. M., Mercer, J. A., Spindel, R. C., Colosi, J. A., Metzger, K., Birdsall, T. G., and Baggeroer, A. B. (1999). "A test of basin-scale acoustic thermometry using a large-aperture vertical array at $3250-\mathrm{km}$ range in the eastern North Pacific Ocean,” J. Acoust. Soc. Am. 105, 3185-3201.

Worcester, P. F., Cornuelle, B. D., Hildebrand, J. A., Hodgkiss, W. S., Jr., Duda, T. F., Boyd, J., Howe, B. M., Mercer, J. A., and Spindel, R. C. (1994). "A comparison of measured and predicted broadband acoustic arrival patterns in travel time-depth coordinates at 1000- $\mathrm{km}$ range," J. Acoust. Soc. Am. 95, 3118-3128. 